\title{
LOCAL SMOOTHING PROPERTIES OF DISPERSIVE EQUATIONS
}

\author{
P. CONSTANTIN AND J. C. SAUT
}

\section{INTRODUCTION}

Is it possible for time evolution partial differential equations which are reversible and conservative to smooth locally the initial data? For the linear wave equation, for instance, the answer is no. However, in [10] T. Kato found a local smoothing property of the Korteweg-de Vries equation: the solution of the initial value problem is, locally, one derivative smoother than the initial datum. Kato's proof uses, in a curcial way, the algebraic properties of the symbol for the Korteweg-de Vries equation and the fact that the underlying spatial dimension is one. Actually, judging from the way several integrations by parts and cancellations conspire to reveal a smoothing effect, one would be inclined to believe this was a special property of the K-dV equation. This is not, however, the case. In this paper, we attempt to describe a general local smoothing effect for dispersive equations and systems. The smoothing effect is due to the dispersive nature of the linear part of the equation. All the physically significant dispersive equations and systems known to us have linear parts displaying this local smoothing property. To mention only a few, the K-dV, Benjamin-Ono, intermediate long wave, various Boussinesq, and Schrödinger equations are included. We study, thus, equations and systems of the form

$$
\begin{gathered}
\partial u / \partial t+i P(D) u=F, \\
u(0, x)=u_{0}(x),
\end{gathered}
$$

where $u=u(t, x), t \in \mathbb{R}, x \in R^{n}, D=\frac{1}{i}\left(\partial / \partial x_{1}, \ldots, \partial / \partial x_{n}\right)$, and $P(D) u$ is defined via a real symbol $p(\xi)$ in the scalar case (or a matrix with real entries in the case of systems),

$$
P(D) u=\int_{\mathbb{R}^{n}} e^{2 i \pi\langle x, \xi\rangle} p(\xi)\left(\mathscr{F}_{2} u\right)(\xi) d \xi,
$$

where $\mathscr{F}_{2}$ is the Fourier transform with respect to the $x$ variables.

Presented at the special session for PDE of the American Mathematical Society meeting in San Antonio, Texas, January 1987; received by the editors August 20, 1987.

1980 Mathematics Subject Classification (1985 Revision). Primary 35Q10.

The first author was partially supported by NSF Grant DMS 860-2031. Sloan Research Fellow. 
The assumptions on $p(\xi)$, reflecting the dispersive nature of $(0.1)$ are that, roughly speaking, $p(\xi)$ behaves like $|\xi|^{m}$ for $|\xi| \rightarrow \infty$, with $m>1$ :

$$
p \in L_{\text {loc }}^{\infty}\left(\mathbb{R}^{n}, \mathbb{R}\right) \text { and is continuously differentiable for }|\xi|>R \text {, }
$$
for some $R \geq 0$.

There exist $m>1, c_{1}>0, c_{2}>0$ such that

$$
|p(\xi)| \leq c_{1}(1+|\xi|)^{m} \text { for all } \xi \in \mathbb{R}^{n}
$$

$\left|\left(\partial p / \partial \xi_{j}\right)(\xi)\right| \geq c_{2}(1+|\xi|)^{m-1}\left|\xi_{j}\right| /|\xi|, \quad$ for all $\xi \in \mathbb{R}^{n},|\xi|>R, j=1, \ldots, n$.

The exact assumptions for systems are a little more complicated since we allow nonselfadjoint matrices $P(D)$. Our assumptions for systems ensure wellposedness of the Cauchy problem in Sobolev spaces; the dispersive character is provided by properties $(0.4),(0.5)$, and $(0.6)$ required to hold for certain combinations of the matrix entries which play the role of propagators (see $\S 4$ for details). In applications, two cases are more frequently encountered. In the first case, $(0.1)$ is of Korteweg-de Vries type, where $u$ is real valued, $n=1$, and $p(\xi)=\xi q\left(\xi^{2}\right)$. This includes the Benjamin-Ono $(p(\xi)=\xi|\xi|)$, intermediate long wave $\left(p(\xi)=\xi^{2} \operatorname{coth} \delta \xi-\xi / \delta, \delta>0\right)$, Smith $\left(p(\xi)=\xi\left(\sqrt{1+\xi^{2}}-1\right)\right)$ equations. In the second case, $(0.2)$ is of Schrödinger type; $u$ is complex valued, $n$ is arbitrary, and $p(\xi)=q\left(|\xi|^{2}\right)$. The systems we treat are various generalizations of water waves systems and systems arising from higher order dispersive equations. In these physical examples the symbol $p$ is related to the dispersion relation between the frequency $\omega$ and the wave number $\xi$ of a plane wave solution $u=e^{i(\omega t-\xi \cdot x)}$. The notion of dispersive waves [18] is that of a wave with a nonlinear dispersion relation, $\omega(\xi) \neq c \cdot \xi$. The cases we treat here would correspond to the situation

$$
\lim _{|\xi| \rightarrow+\infty}|\omega(\xi)| /|\xi|=+\infty
$$

The equation $(0.1)$ is conservative and time reversible: the solution defines a continuous unitary group on every Sobolev space $H^{s}\left(\mathbb{R}^{n}\right)$. (In the case of nonselfadjoint systems, our assumptions imply that the Sobolev norms of the solutions are controlled for all (positive or negative) times by those of the initial data.) Thus a global smoothing effect is excluded in Sobolev spaces.

A typical result that we obtain is: if $u_{0}$ belongs to $H^{s}\left(\mathbb{R}^{n}\right)$ then, for almost every $t \neq 0$, the solution $u(t, \cdot)$ belongs to $H_{\mathrm{loc}}^{s+d}\left(\mathbb{R}^{n}\right)$ where $d=(m-1) / 2$; that is, $d$ depends on the order of the operator $P(D)$ but not on the spatial dimension $n$. The higher the order $m$, the more dispersive the equation is and stronger the local smoothing effect becomes.

For nonlinear equations one needs to measure the smoothing effect in terms of other Sobolev norms than those based on $L^{2}$. In that case, our results depend on the dimension $n$. The proofs rely on a new restriction lemma for 
Fourier transform $(\S 1)$. The smoothing effect is proven for $F=0$ in a quite straightforward manner, using the growth of $p(\xi)$ at infinity and the restriction lemma $(\S 2)$. In $\S 3$ we prove the local smoothing property in the nonhomogeneous case $(F \neq 0)$; as an application we discuss Schrödinger equations with various potentials. $\S 4$ is devoted to the study of systems. Two typical examples of systems that we treat are (see the concrete Examples 4.1 in the text)

$$
\left\{\begin{array}{l}
u_{t}-\beta_{1} H_{1} u_{x}-\beta_{1} H_{2} v_{x}=0, \quad x \in \mathbb{R}, t \in \mathbb{R}, \\
v_{t}-\beta_{2} H_{3} v_{x}-\beta_{2} H_{2} u_{x}=0,
\end{array}\right.
$$

where $H_{i}, i=1,2,3$, are nonlocal operators, $H_{2}$ being a smoothing operator, and the systems arising from equations of the form

$$
\partial^{2} u / \partial t^{2}+(-1)^{k} \Delta^{k} u=0, \quad k \geq 2 .
$$

We give in $\S 5$ a few applications to nonlinear problems. The local smoothing property for nonlinear problems can be used to either derive existence results for rough initial data or to prove regularity results. We present here only those nonlinear examples whose treatment follows directly from that of their linear parts. Other cases will be considered elsewhere.

While global smoothing cannot occur in $L^{2}$-Sobolev spaces, there exists the possibility, mostly for linear equations, of global smoothing in different spaces. Thus Hayashi and Nakamizu and Tsutsumi $[8,9]$ prove a global smoothing property for a class of nonlinear Schrödinger equation, assuming the initial data decays sufficiently at $\infty$. Under the assumption of initial data in $L^{1}\left(\mathbb{R}^{n}\right)$, Balabane and Emami Rad [2,3] obtain a global smoothing in Sobolev $W^{p, k}$ spaces for linear equations of Schrödinger type of high enough order provided the spatial dimension is correspondingly large enough.

Results which overlap with some of our results were obtained independently by Sjölin [15]. Indeed one of the inequalities he uses in [15] amounts to a local smoothing property for the free Schrodinger equation, essentially the same as the one we obtain for that equation. His work is concerned with a problem of interest in harmonic analysis. We wish to thank C. Sogge for bringing Sjölin's work to our attention and for stimulating discussions. Part of the results we present here were announced in a Note aux Comptes Rendus [5].

We conclude this introduction by establishing the notation used in the paper.

The norm in $L^{q}\left(\mathbb{R}^{n}\right), 1 \leq q \leq+\infty$, will be denoted by $\|\cdot\|_{q} ;\|\cdot\|_{p, q}$ will stand for the norm in $L^{p}\left(\mathbb{R}, L^{q}\left(\mathbb{R}^{n}\right)\right) \quad(1 \leq p, q \leq+\infty)$. For a function $f$ of $t, x \quad\left(t \in \mathbb{R}, x \in \mathbb{R}^{n}\right)$, we use $\mathscr{F}_{2} f$ or $\hat{f}$ for the Fourier transform with respect to $x$, and $\mathscr{F} f$ for the Fourier transform with respect to both variables $(t, x)$. For $s \in \mathbb{R}$, the classical Sobolev space $H^{s}\left(\mathbb{R}^{n}\right)$ is defined as the set of $v \in \mathscr{S}^{\prime}\left(\mathbb{R}^{n}\right)$ (the tempered distributions) such that $\left(1+|\xi|^{2}\right)^{s / 2} \hat{v} \in L^{2}\left(\mathbb{R}^{n}\right)$, equipped with the norm $\left[\int_{\mathbb{R}^{n}}\left(1+|\xi|^{2}\right)^{s}|\hat{v}(\xi)|^{2} d \xi\right]^{1 / 2}$. Also we set

$$
\begin{aligned}
H_{u ; \text { loc }}^{s}\left(\mathbb{R}^{n}\right)=\left\{v \in \mathscr{S}^{\prime}\left(\mathbb{R}^{n}\right), \forall \chi \in C_{0}^{\infty}\left(\mathbb{R}^{n}\right), \exists C_{\chi}>0, \chi v \in H^{s}\left(\mathbb{R}^{n}\right)\right. \\
\text { with } \left.\left\|\left(\tau_{a} \chi\right) v\right\|_{H^{s}\left(\mathbb{R}^{n}\right)} \leq C_{\chi}, \forall a \in \mathbb{R}^{n}\right\}
\end{aligned}
$$

(where $\tau_{a} \chi(x)=\chi(x-a)$ ). 
We also use the potential spaces $H^{s, p}\left(\mathbb{R}^{n}\right), s \in \mathbb{R}, 1<p<\infty$,

$$
\begin{aligned}
H^{s, p}\left(\mathbb{R}^{n}\right) & =\left\{f \in \mathscr{S}^{\prime}\left(\mathbb{R}^{n}\right), \mathscr{F}^{-1}\left(1+|\xi|^{2}\right)^{s / 2} \mathscr{F} f \in L^{p}\left(\mathbb{R}^{n}\right)\right\} \\
& =\left\{f \in \mathscr{S}^{\prime}\left(\mathbb{R}^{n}\right),(I-\Delta)^{s / 2} f \in L^{p}\left(\mathbb{R}^{n}\right)\right\},
\end{aligned}
$$

equipped with the norm

$$
\|f\|_{H^{s, p}\left(\mathbb{R}^{n}\right)}=\left\|(1-\Delta)^{s / 2} f\right\|_{p} .
$$

$H_{u ; \text { loc }}^{s, p}\left(\mathbb{R}^{n}\right)$ is defined as above.

1. A LEMMA ON THE RESTRICTION OF THE FOURIER TRANSFORM

We introduce $\chi: \mathbb{R}^{n+1} \rightarrow \mathbb{R}$ of the type

$$
\chi(t, x)=\chi_{0}(t) \chi_{1}\left(x_{1}\right) \cdots \chi_{n}\left(x_{n}\right), \quad \text { where } x=\left(x_{1}, \ldots, x_{n}\right)
$$

and

$$
\chi_{j} \in C_{0}^{\infty}(\mathbb{R}), \quad j=0,1, \ldots, n .
$$

Also let a real $q, 1 \leq q \leq 2$, and a real $\alpha$ be such that

$$
\begin{cases}2 \alpha<m-1-((2-q) / q) n, & \text { if } 1 \leq q<2 \\ 2 \alpha \leq m-1, & \text { if } q=2\end{cases}
$$

We can now state the main result of this section.

Theorem 1.1. Assume that $p$ satisfies (0.4), (0.6) if $q=2$ and also, (0.5) if $1 \leq q<2$. Let $\alpha$ be given by (1.1). Then there exists a constant $C_{\chi}$ depending only on $\chi, q, \alpha, n, m$ such that for every $f \in \mathscr{S}\left(\mathbb{R}^{n+1}\right)$ (rapidly decaying, smooth functions )

$$
\left(\int_{\mathbb{R}^{n}}\left(1+|\xi|^{2}\right)^{\alpha q / 2}|(\mathscr{F} \chi f)(p(\xi), \xi)|^{q} d \xi\right)^{1 / q} \leq C_{\chi}\|f\|_{L^{2}\left(\mathbb{R}^{n+1}\right)} .
$$

Moreover, the constant $C_{\chi}$ can be estimated as follows:

$$
C_{\chi} \leq C\left[\prod_{j=0}^{n}\left\|\chi_{j}\right\|_{2}+\left\|\chi_{0}\right\|_{\infty} \sum_{j=1}^{n}\left\|\chi_{j}\right\|_{2}\left\|\chi_{1}\right\|_{\infty} \cdots\left\|\tilde{\chi}_{j}\right\|_{\infty} \cdots\left\|\chi_{n}\right\|_{\infty}\right]
$$

where $\sim$ means that the corresponding term is omitted, and $C$ is an absolute constant.

Proof of Theorem 1.1. We break the integral in (1.2) into

$$
\int_{|\xi| \leq R}+\int_{|\xi|>R}(\text { where } R \text { is as in }(0.4)-(0.6)) \text {. }
$$

The first integral can be easily bounded

$$
\begin{aligned}
\int_{|\xi| \leq R}(1 & \left.+|\xi|^{2}\right)^{\alpha q / 2}|\mathscr{F}(\chi f)(p(\xi), \xi)|^{q} d \xi \leq C_{R}\|\mathscr{F}(\chi f)\|_{L^{\infty}\left(\mathbb{R}^{n+1}\right)}^{q} \\
& \leq C_{R}\|\chi f\|_{L^{1}\left(\mathbb{R}^{n+1}\right)}^{q} \\
& \leq C_{R}\left\|\chi_{0}\right\|_{2}^{q} \cdots\left\|\chi_{n}\right\|_{2}^{q}\|f\|_{L^{2}\left(\mathbb{R}^{n+1}\right)}^{q} .
\end{aligned}
$$


We need to estimate $\int_{|\xi|>R}$. Let $\Gamma_{j}=\left\{\xi \in \mathbb{R}^{n},|\xi|>R,\left|\xi_{j}\right| \geq(1 / \sqrt{n})|\xi|\right\}$ for $j=1, \ldots, n$. It is clear that $\left\{\xi \in \mathbb{R}^{n},|\xi|>R\right\} \subset \bigcup_{j=1}^{n} \Gamma_{j}$. Therefore it is sufficient to estimate each integral over $\Gamma_{j}$. In order to simplify the exposition, we choose $j=1$. We need thus to estimate

$$
\int_{\Gamma_{1}}\left(1+|\xi|^{2}\right)^{\alpha q / 2}|\mathscr{F}(\chi f)(p(\xi), \xi)|^{q} d \xi .
$$

We perform a change of variables $\xi \rightarrow \eta(\xi)$ defined by

$$
\eta: \Gamma_{1} \rightarrow \mathbb{R}^{n}, \quad \eta_{1}(\xi)=p(\xi), \eta_{j}(\xi)=\xi_{j}, j=2, \ldots, n
$$

The Jacobian of the transformation $\eta$ is

$$
\operatorname{det}\left(\left(\partial \eta_{i} / \partial \xi_{j}\right)(\xi)\right)=\left(\partial p / \partial \xi_{1}\right)(\xi)
$$

In view of $(0.6)$, for $\xi \in \Gamma_{1}$

$$
\begin{aligned}
\left|\operatorname{det}\left(\partial \eta_{i} / \partial \xi_{j}\right)(\xi)\right| \geq C_{2}(1+|\xi|)^{m-1}\left|\xi_{1}\right| /|\xi| & \geq\left(C_{2} / \sqrt{n}\right)(1+|\xi|)^{m-1} \\
& \geq\left(C_{3} / \sqrt{n}\right)\left(1+|\xi|^{2}\right)^{(m-1) / 2}
\end{aligned}
$$

The set $\Gamma_{1}$ has two connected components

$$
\Gamma_{1}^{ \pm}=\left\{\xi \in \Gamma_{1}, \xi_{1} \gtrless 0\right\}
$$

On each of these components, the map $\eta: \Gamma_{1}^{ \pm} \rightarrow \mathbb{R}^{n}$ is one-to-one. Indeed, if $\eta(\xi)=\eta(\tilde{\xi})$, it follows that $\xi_{j}=\tilde{\xi}_{j}$ for $j \geq 2$ and $p\left(\xi_{1}, \xi_{2}, \ldots, \xi_{n}\right)=$ $p\left(\tilde{\xi}_{1}, \xi_{2}, \ldots, \xi_{n}\right)$. Since $\left(\partial p / \partial \xi_{1}\right)(\xi) \neq 0$ for $\xi \in \Gamma_{1}^{ \pm}$and since the segment joining $\left(\xi_{1}, \xi_{2}, \ldots, \xi_{n}\right)$ to $\left(\tilde{\xi}_{1}, \xi_{2}, \ldots, \xi_{n}\right)$ lies in $\Gamma_{1}^{ \pm}$, it follows that $\xi_{1}=\tilde{\xi}_{1}$.

Let us denote by $G_{1}^{ \pm}$the images of $\Gamma_{1}^{ \pm}$and by $\xi^{ \pm}$the inverses, $\xi^{ \pm}: G_{1}^{ \pm} \rightarrow$ $\Gamma_{1}^{ \pm}$. We have

$$
\begin{aligned}
& \int_{\Gamma_{1}^{ \pm}}\left(1+|\xi|^{2}\right)^{q \alpha / 2}|\mathscr{F}(\chi f)(p(\xi), \xi)|^{q} d \xi \\
& \quad=\int_{G_{1}^{ \pm}}\left(1+\left|\xi^{ \pm}(\eta)\right|^{q \alpha / 2}\left|\operatorname{det}(\partial \eta / \partial \xi)\left(\xi^{ \pm}(\eta)\right)\right|^{-1}\left|\mathscr{F}(\chi f)\left(\eta_{1}, \xi_{1}^{ \pm}(\eta), \eta^{\prime}\right)\right|^{q} d \eta .\right.
\end{aligned}
$$

We denoted $\eta^{\prime}=\left(\eta_{2}, \ldots, \eta_{n}\right)$ and $\xi_{1}^{ \pm}(\eta)$ is the first component of $\xi^{ \pm}(\eta)$. From (1.5) we deduce

$$
\left(1+|\xi|^{2}\right)^{q \alpha / 2}|\operatorname{det}(\partial \eta / \partial \xi)(\xi)|^{-1} \leq\left(\sqrt{n} / C_{3}\right)\left(1+|\xi|^{2}\right)^{-(m-1-q \alpha) / 2} .
$$

Thus

$$
\begin{aligned}
& \int_{\Gamma_{1}^{ \pm}}\left(1+|\xi|^{2}\right)^{q \alpha / 2}|\mathscr{F}(\chi f)(p(\xi), \xi)|^{q} d \xi \\
& \quad \leq\left(\sqrt{n} / C_{3}\right) \int_{G_{1}^{ \pm}}\left(1+\left|\xi^{ \pm}(\eta)\right|^{2}\right)^{-(m-1-q \alpha) / 2}\left|\mathscr{F}(\chi f)\left(\eta_{1}, \xi_{1}^{ \pm}(\eta), \eta^{\prime}\right)\right|^{q} d \eta_{1} d \eta^{\prime}
\end{aligned}
$$

Let us note that since $\xi_{j}^{ \pm}(\eta)=\eta_{j}$ for $j=2, \ldots, n$, it follows that

$$
\left|\xi^{ \pm}(\eta)\right| \geq\left|\eta^{\prime}\right| \text {. }
$$


Also, since $p\left(\xi^{ \pm}(\eta)\right)=\eta_{1}$, from $(0.5)$ we infer that

$$
\left|\eta_{1}\right| \leq C_{1}\left(1+\left|\xi^{ \pm}(\eta)\right|\right)^{m} \leq C_{1}^{\prime}\left(1+\left|\xi^{ \pm}(\eta)\right|^{2}\right)^{m / 2} .
$$

Assuming with no loss of generality $C_{1}^{\prime} \geq 1$ we have thus

$$
\left(1+\left|\eta_{1}\right|^{2}\right)^{1 / 2} \leq 2 C_{1}^{\prime}\left(1+\left|\xi^{ \pm}(\eta)\right|^{2}\right)^{m / 2} .
$$

Using Hölder's inequality we have

$$
\begin{aligned}
\int_{G_{1}^{ \pm}}(1 & \left.+\left|\xi^{ \pm}(\eta)\right|^{2}\right)^{-(m-1-q \alpha) / 2}\left|\mathscr{F}(\chi f)\left(\eta_{1}, \xi_{1}^{ \pm}(\eta), \eta^{\prime}\right)\right|^{q} d \eta \\
\leq & {\left[\int_{G_{1}^{ \pm}}\left(1+\left|\xi^{ \pm}(\eta)\right|^{2}\right)^{-(m-1-q \alpha) /(2-q)} d \eta\right]^{(1-q) / 2} } \\
& \times\left[\int_{G_{1}^{ \pm}}\left|\mathscr{F}(\chi f)\left(\eta_{1}, \xi_{1}^{ \pm}(\eta), \eta^{\prime}\right)\right|^{2} d \eta\right]^{q / 2} .
\end{aligned}
$$

First we claim that $\int_{G_{1}^{ \pm}}\left(1+\left|\xi^{ \pm}(\eta)\right|^{2}\right)^{-(m-1-q \alpha) /(2-q)} d \eta$ is finite (in the case $q=2$ the claim is that $\left(1+\left|\xi^{ \pm}(\eta)\right|^{2}\right)^{-(m-1-2 \alpha) / 2}$ is uniformly bounded for $\eta \in G_{1}^{ \pm}$, which is obvious in view of $\left.(1.1)\right)$.

Indeed for $q<2,(1.1)$ is equivalent to

$$
(2 /(2-q))(m-1-q \alpha)>m+n-1 .
$$

Therefore there exists $\delta>0$ such that

$$
(2 /(2-q))(m-1-q \alpha)=m(1+\delta)+(n-1)(1+\delta) .
$$

Writing

$$
\left(1+\left|\xi^{ \pm}(\eta)\right|^{2}\right)^{-(m-1-q \alpha) /(2-q)}=\left(1+\left|\xi^{ \pm}(\eta)\right|^{2}\right)^{-m(1+\delta) / 2}\left(1+\left|\xi^{ \pm}(\eta)\right|^{2}\right)^{-(n-1)(1+\delta) / 2}
$$

we obtain, using (1.6) and (1.7)

$$
\left(1+\left|\xi^{ \pm}(\eta)\right|^{2}\right)^{-(m-1-q \alpha) /(2-q)} \leq C\left(1+\left|\eta_{1}\right|^{2}\right)^{-(1+\delta) / 2}\left(1+\left|\eta^{\prime}\right|^{2}\right)^{-(n-1)(1+\delta) / 2}
$$

and thus

$$
\begin{aligned}
\int_{G_{1}^{ \pm}} & \left(1+\left|\xi^{ \pm}(\eta)\right|^{2}\right)^{-(m-1-q \alpha) /(2-q)} d \eta \\
& \leq C \int_{\mathbb{R}^{n}}\left(1+\left|\eta_{1}\right|^{2}\right)^{-(1+\delta) / 2}\left(1+\left|\eta^{\prime}\right|^{2}\right)^{-(n-1)(1+\delta) / 2} d \eta<+\infty
\end{aligned}
$$

We claim now that

$$
\int_{G_{1}^{ \pm}}\left|\mathscr{F}(\chi f)\left(\eta_{1}, \xi_{1}^{ \pm}(\eta), \eta^{\prime}\right)\right|^{2} d \eta \leq C_{\chi}^{2}\|f\|_{L^{2}\left(\mathbb{R}^{n+1}\right)}^{2} .
$$

Actually, more is true, that is

$$
\int_{\mathbb{R}^{n}} \operatorname{Sup}_{\xi_{1} \in \mathbb{R}}\left|\mathscr{F}(\chi f)\left(\eta_{1}, \xi_{1}, \eta^{\prime}\right)\right|^{2} d \eta \leq C_{\chi}^{2}\|f\|_{L^{2}\left(\mathbb{R}^{n+1}\right)}^{2} .
$$


Indeed we write $\chi f$ as

$$
\chi f=\chi_{1}\left(x_{1}\right)(\tilde{\chi} f)\left(t, x_{1}, x_{2}, \ldots, x_{n}\right)
$$

with $\tilde{\chi}=\chi_{0} \chi_{2} \cdots \chi_{n}$. Then

$$
\mathscr{F}(\chi f)\left(\eta_{1}, \xi_{1}, \eta^{\prime}\right)=\int_{-\infty}^{\infty} \hat{\chi}_{1}\left(\xi_{1}-x\right) \mathscr{F}(\tilde{\chi} f)\left(\eta_{1}, x, \eta^{\prime}\right) d x
$$

This yields

$$
\left|\mathscr{F}(\chi f)\left(\eta_{1}, \xi_{1}, \eta^{\prime}\right)\right|^{2} \leq\left\|\chi_{1}\right\|_{2}^{2} \int_{-\infty}^{\infty} \mathscr{F}\left|(\tilde{\chi} f)\left(\eta_{1}, x, \eta^{\prime}\right)\right|^{2} d x .
$$

The right-hand side is clearly independent of $\xi_{1}$. Taking the supremum and integrating, it follows that

$$
\begin{aligned}
\int_{\mathbb{R}^{n}} \operatorname{Sup}_{\xi_{1}} & \left|\mathscr{F}(\chi f)\left(\eta_{1}, \xi_{1}, \eta^{\prime}\right)\right|^{2} d \eta \\
& \leq\left\|\chi_{1}\right\|_{2}^{2} \int_{\mathbb{R}^{n+1}}\left|\mathscr{F}(\tilde{\chi} f)\left(\eta_{1}, x, \eta^{\prime}\right)\right|^{2} d \eta_{1} d x d \eta^{\prime} \\
& =\left\|\chi_{1}\right\|_{2}^{2}\|\tilde{\chi} f\|_{L^{2}\left(\mathbb{R}^{n+1}\right)}^{2} \\
& \leq\left\|\chi_{1}\right\|_{2}^{2}\|\tilde{\chi}\|_{\infty}^{2}\|f\|_{L^{2}\left(\mathbb{R}^{n+1}\right)}^{2} .
\end{aligned}
$$

This concludes the proof of Theorem 1.1, with the constant $C_{\chi}$ estimated by (1.3). If $q=2$ we notice that condition (0.5) is not needed. It was used only to provide decay in $\left|\eta_{i}\right|, i=1, \ldots, n$.

We conclude this section with various remarks and variants of Theorem 1.1.

Remark 1.1 (nonisotropic symbols). Conditions (0.5) and (1.1) can be replaced by

$$
\begin{gathered}
|p(\xi)| \leq C(1+|\xi|)^{m^{\prime}}, \quad m^{\prime} \geq m, \\
\left\{\begin{array}{l}
2 \alpha<m^{\prime}-1-((2-q) / q) n-(2 / q)\left(m^{\prime}-m\right), \quad \text { if } q<2, \\
2 \alpha \leq m-1, \quad \text { if } q=2 .
\end{array}\right.
\end{gathered}
$$

This is easily checked by an inspection of the proof of Theorem 1.1. For instance, if $n=2$ an $p\left(\xi_{1}, \xi_{2}\right)=\xi_{1}^{2}+\xi_{2}^{4}$, then $m=2$ and $m^{\prime}=4$. The condition $(1.1)^{\prime}$ implies that for $q$ belonging to $\left(\frac{8}{5}, 2\right]$, one obtains some degree of local smoothing, $\alpha>0$. Of course we obtain better results in the $\xi_{2}$ direction by choosing adapted anisotropic weights $\left(1+\left|\xi_{1}\right|^{2}\right)^{\alpha q / 2}\left(1+\left|\xi_{2}\right|^{2}\right)^{\beta q / 2}$. We will not proceed further in this direction.

Remark 1.2 (an $L^{r^{\prime}}$ estimate $1 \leq r^{\prime}<2$ ). In the following remark we show how one can adapt the proof of Theorem 1.1 in order to get an $L^{r^{\prime}}, 1 \leq r^{\prime}<2$, estimate. These types of estimates are useful in nonlinear problems. We are still given $\chi \in C_{0}^{\infty}\left(\mathbb{R}^{n+1}\right)$ as above and $1 \leq q<2$. Furthermore, let $r$ be a real $\geq 2$. Instead of $(1.1)$ we assume that $\alpha>0$ is given satisfying

$$
\left\{\begin{array}{l}
\alpha<(m-1) / r-n(1 / q-1 / r), \quad r \geq 2, r>q, \\
\alpha \leq(m-1) / 2, \quad \text { if } r=q=2 .
\end{array}\right.
$$


Then there exists $C_{\chi}^{\prime}>0$ (see below) such that for every $f \in \mathscr{S}\left(\mathbb{R}^{n+1}\right)$,

$$
\left[\int_{\mathbb{R}^{n}}\left(1+|\xi|^{2}\right)^{\alpha q / 2}|\mathscr{F}(\chi f)(p(\xi), \xi)|^{q} d \xi\right]^{1 / q} \leq C_{\chi}^{\prime}\|f\|_{L^{r^{\prime}}\left(\mathbb{R}^{n+1}\right)}
$$

where $1 / r+1 / r^{\prime}=1$.

The proof of (1.13) follows that of Theorem 1.1. Let us briefly indicate the main differences. First, instead of (1.8) we obtain, still with Hölder's inequality,

$$
\begin{aligned}
\int_{G_{1}^{ \pm}}(1 & \left.+\left|\xi^{ \pm}(\eta)\right|^{2}\right)^{-(m-1-q \alpha) / 2}\left|\mathscr{F}(\chi f)\left(\eta_{1}, \xi_{1}^{ \pm}(\eta), \eta^{\prime}\right)\right|^{q} d \eta \\
\leq & {\left[\int_{G_{1}^{ \pm}}\left(1+\left|\xi^{ \pm}(\eta)\right|^{2}\right)^{-((m-1-q \alpha) / 2)(r /(r-q))} d \eta\right]^{1-q / r} } \\
& \times\left[\int_{G_{1}^{ \pm}}\left|\mathscr{F}(\chi f)\left(\eta_{1}, \xi_{1}^{ \pm}(\eta), \eta^{\prime}\right)\right|^{r} d \eta\right]^{q / r} .
\end{aligned}
$$

We claim that

$$
\int_{G_{1}^{ \pm}}\left(1+\left|\xi^{ \pm}(\eta)\right|^{2}\right)^{-((m-1-q \alpha) / 2)(r /(r-q))} d \eta \quad \text { is finite. }
$$

(In the case $q=r=2$ the claim is that $\left(1+\left|\xi^{ \pm}(\eta)\right|^{2}\right)^{-(m-1-2 \alpha) / 2}$ is uniformly bounded for $\eta \in G_{1}^{ \pm}$, which is obvious in view of (1.12) and which is a case included in Theorem 1.1.)

Indeed for $r>q(1.12)$ is equivalent to

$$
(r /(r-q))(m-1-q \alpha)>m+n-1
$$

and we can then proceed as in the derivation of (1.9).

It remains to show that

$$
\int_{G_{1}^{ \pm}}\left|\mathscr{F}(\chi f)\left(\eta_{1}, \xi_{1}^{ \pm}(\eta), \eta^{\prime}\right)\right|^{r} d \eta \leq C_{\chi}^{r}\|f\|_{L^{\prime}\left(\mathbb{R}^{n+1}\right)}^{r} .
$$

As in the $L^{2}$ case, we prove more; namely,

$$
\int_{\mathbb{R}^{n}} \operatorname{Sup}_{\xi_{1} \in \mathbb{R}}\left|\mathscr{F}(\chi f)\left(\eta_{1}, \xi_{1}, \eta^{\prime}\right)\right|^{r} d \eta \leq C_{\chi}^{r}\|f\|_{L^{r^{\prime}\left(\mathbb{R}^{n+1}\right)}}^{r} .
$$

Proceeding as in the derivation of (1.11), we arrive at

$$
\begin{aligned}
\left|\mathscr{F}(\chi f)\left(\eta_{1}, \xi_{1}, \eta^{\prime}\right)\right|^{r} & =\left|\int_{-\infty}^{\infty} \hat{\chi}_{1}\left(\xi_{1}-x\right) \mathscr{F}(\tilde{\chi} f)\left(\eta_{1}, x, \eta^{\prime}\right) d x\right|^{r} \\
& \leq\left\|\hat{\chi}_{1}\right\|_{L^{\prime}(\mathbb{R})}^{r} \int_{-\infty}^{\infty}\left|\mathscr{F}(\tilde{\chi} f)\left(\eta_{1}, x, \eta^{\prime}\right)\right|^{r} d x .
\end{aligned}
$$

Taking the supremum and integrating yields

$$
\begin{aligned}
& \int_{\mathbb{R}^{n}} \operatorname{Sup}_{\xi_{1}}\left|\mathscr{F}(\chi f)\left(\eta_{1}, \xi_{1}, \eta^{\prime}\right)\right|^{r} d \eta \\
& \quad \leq\left\|\hat{\chi}_{1}\right\|_{L^{r^{\prime}}(\mathbb{R})}^{r} \int_{\mathbb{R}^{n+1}}\left|\mathscr{F}(\tilde{\chi} f)\left(\eta_{1}, x, \eta^{\prime}\right)\right|^{r} d \eta_{1} d x d \eta^{\prime}
\end{aligned}
$$


and by the Hausdorff-Young theorem

$$
\begin{aligned}
\int_{\mathbb{R}^{n}} \operatorname{Sup}_{\xi_{1}}\left|\mathscr{F}(\chi f)\left(\eta_{1}, \xi_{1}, \eta^{\prime}\right)\right|^{r} d \eta & \leq\left\|\hat{\chi}_{1}\right\|_{L^{r^{\prime}(\mathbb{R})}}^{r}\|\tilde{\chi} f\|_{L^{r^{\prime}}\left(\mathbb{R}^{n+1}\right)}^{r} \\
& \leq C\left\|\hat{\chi}_{1}\right\|_{L^{r^{\prime}(\mathbb{R})}}^{r}\|\tilde{\chi}\|_{\infty}^{r}\|f\|_{L^{r^{\prime}\left(\mathbb{R}^{n+1}\right)}}^{r}
\end{aligned}
$$

and this gives obviously (1.13) with a constant $C_{\chi}^{\prime}$ which can be estimated as follows (where $C$ is an absolute constant):

$$
C_{\chi}^{\prime} \leq C\left[\left\|\chi_{0}\right\|_{r} \cdots\left\|\chi_{n}\right\|_{r}+\left\|\chi_{0}\right\|_{\infty} \sum_{j=1}^{n}\left\|\hat{\chi}_{j}\right\|_{r^{\prime}}\left\|\chi_{1}\right\|_{\infty} \cdots\left\|\tilde{\chi}_{j}\right\|_{\infty} \cdots\left\|\chi_{n}\right\|_{\infty}\right]
$$

(the term $\left\|\chi_{0}\right\|_{r} \cdots\left\|\chi_{n}\right\|_{r}$ comes from the equivalent of (1.4)).

In the case $n=1$ one can dispense with time localization if condition (0.6) is fulfilled for any $|\xi|>0$. This allows us to obtain global in time, local in space smoothing (see $\S 2$ ). We present briefly the argument in the next remark.

Remark 1.3. Let $2 \leq \beta \leq+\infty, 1 / \beta+1 / \beta^{\prime}=1$. Then there exists an absolute constant $C>0$ such that for every $a \in \mathscr{S}\left(\mathbb{R}^{2}\right), \chi \in C_{0}^{\infty}(\mathbb{R})$

$$
\begin{aligned}
& {\left[\int_{\mathbb{R}}|\xi|^{m-1}|\mathscr{F}(\chi a)(p(\xi), \xi)|^{\beta} d \xi\right]^{1 / \beta}} \\
& \quad \leq C\left(\|\chi\|_{\beta}+\|\hat{\chi}\|_{\beta^{\prime}}\right)\left(\|a\|_{\beta^{\prime}, \beta^{\prime}}+\|a\|_{1, \beta^{\prime}}\right) .
\end{aligned}
$$

Moreover, if one takes $R=0(0.6)$ (which is the case of the examples mentioned in the Introduction), the right-hand side of (1.17) can be replaced by

$$
C\left(\|\chi\|_{\beta}+\|\hat{\chi}\|_{\beta^{\prime}}\right)\|a\|_{\beta^{\prime} \beta^{\prime}} .
$$

In fact, integrating over $\{|\xi| \leq R\}$ gives

$$
\left[\int_{|\xi| \leq R}|\xi|^{m-1}|\mathscr{F}(\chi a)(p(\xi), \xi)|^{\beta} d \xi\right]^{1 / \beta} \leq C_{R}\|\chi\|_{\beta}\|a\|_{1, \beta^{\prime}}
$$

To estimate $\int_{|\xi| \geq R}$, we first bound $\operatorname{Sup}_{\xi \in \mathbb{R}}|\mathscr{F}(\chi a)(\tau, \xi)|$. One has

$$
\begin{aligned}
|\mathscr{F}(\chi a)(\tau, \xi)| & =\left|\int_{\mathbb{R}} e^{-2 i \pi t \tau}\left[\int_{\mathbb{R}} e^{-2 i \pi x \xi} \chi(x) a(t, x) d x\right] d t\right| \\
& =\left|\int_{\mathbb{R}} e^{-2 i \pi t \tau} \int_{\mathbb{R}} \hat{\chi}(\eta)(\mathscr{F} a)(t, \xi-\eta) d \eta d t\right| \\
& =\left|\int_{\mathbb{R}} \hat{\chi}(\eta)(\mathscr{F} a)(\tau, \xi-\eta) d \eta\right| \\
& \leq\|\hat{\chi}\|_{\beta^{\prime}}\left(\int_{\mathbb{R}}|\mathscr{F} a|^{\beta}(\tau, \eta) d \eta\right)^{1 / \beta}
\end{aligned}
$$

and

$$
\operatorname{Sup}_{\xi \in \mathbb{R}}|\mathscr{F}(\chi a)(\tau, \xi)|^{\beta} \leq\|\hat{\chi}\|_{\beta^{\prime}}^{\beta} \int_{\mathbb{R}}|\mathscr{F} a|^{\beta}(\tau, \eta) d \eta
$$


Therefore

$$
\begin{aligned}
\int_{|\xi| \geq R}|\mathscr{F}(\chi a)(p(\xi), \xi)|^{\beta}|\xi|^{m-1} d \xi & \leq \int_{|\xi| \geq R} \operatorname{Sup}_{\eta \in \mathbb{R}}|\mathscr{F}(\chi a)(p(\xi), \eta)|^{\beta}|\xi|^{m-1} d \xi \\
& \leq\|\hat{\chi}\|_{\beta^{\prime}}^{\beta} \int_{|\xi| \geq R} \int_{\mathbb{R}}|\mathscr{F} a|^{\beta}(p(\xi), \eta)|\xi|^{m-1} d \xi d \eta \\
& \leq C\|\hat{\chi}\|_{\beta^{\prime}}^{\beta} \int_{|\xi| \geq R} \int_{\mathbb{R}}|\mathscr{F} a|^{\beta}(p(\xi), \eta)\left|p^{\prime}(\xi)\right| d \xi d \eta .
\end{aligned}
$$

Using the change of variables $\eta=p(\xi)$ (see proof of Theorem 1.1) we finally arrive at

$$
\int_{|\xi| \geq R}|\mathscr{F}(\chi a)(p(\xi), \xi)|^{\beta}|\xi|^{m-1} d \xi \leq C\|\hat{\chi}\|_{\beta^{\prime}}^{\beta}\|a\|_{\beta^{\prime}, \beta^{\prime}}^{\beta}
$$

Regrouping (1.19) and (1.20) yields

$$
\begin{aligned}
& {\left[\int|\mathscr{F}(\chi a)(p(\xi), \xi)|^{\beta}|\xi|^{m-1} d \xi\right]^{1 / \beta}} \\
& \quad \leq C\left(\|\chi\|_{\beta}+\|\hat{\chi}\|_{\beta^{\prime}}\right)\left(\|a\|_{\beta^{\prime}, \beta^{\prime}}+\|a\|_{1, \beta^{\prime}}\right) .
\end{aligned}
$$

The right-hand side of (1.21) reduces to $C\left(\|\chi\|_{\beta}+\|\hat{\chi}\|_{\beta^{\prime}}\right)\|a\|_{\beta^{\prime}, \beta^{\prime}}$ when $R=0$.

\section{THE SMOOTHING PROPERTY: SCALAR CASE}

The main result of this section is

Theorem 2.1. We assume that $p$ satisfies (0.4), and (0.6). Let $s \geq-(m-1) / 2$, $u_{0} \in H^{s}\left(\mathbb{R}^{n}\right)$. Then for $\chi \in C_{0}^{\infty}\left(\mathbb{R}^{n+1}\right)$ of the form given in Theorem 1.1 the solution $u$ of $(0,1)$ associated to $u_{0}$ satisfies

$$
\int_{\mathbb{R}^{n+1}} \chi^{2}(t, x)\left|(I-\Delta)^{(m-1+2 s) / 4} u(t, x)\right|^{2} d x d t \leq C_{\chi}^{2}\left\|u_{0}\right\|_{H^{s}\left(\mathbb{R}^{n}\right)}^{2},
$$

where $C_{\chi}$ is the constant given by (1.3). In particular,

$$
u \in L^{2}\left(-T, T ; H_{u ; \operatorname{loc}}^{s+(m-1) / 2}\left(\mathbb{R}^{n}\right)\right)
$$

for every $T>0$.

Remark 2.1. It follows from Theorem 2.1 and the Sobolev imbedding theorem that $u \in L^{2}\left(-T, T ; C_{b}^{k}\left(\mathbb{R}^{n}\right)\right)$ where $C_{b}^{k}\left(\mathbb{R}^{n}\right)$ is the space of $C^{k}$ functions with bounded derivatives up to order $k$ provided $s>(n-m+2 k+1) / 2$.

Proof of Theorem 2.1. We proceed by duality. Let $f \in \mathscr{S}\left(\mathbb{R}^{n+1}\right)$. We set

$$
I=\left|\int_{\mathbb{R}^{n+1}} \chi(t, x)(I-\Delta)^{(m-1+2 s) / 4} u(t, x) \overline{f(t, x)} d t d x\right| .
$$

Since $u(t, \cdot)=e^{-i t P(D)} u_{0}$, we can write by transposition

$$
I=\left|\left\langle u_{0},(I-\Delta)^{(m-1+2 s) / 4} \int_{\mathbb{R}} e^{i t P(D)}(\chi f) d t\right\rangle\right|
$$


where $\langle F, G\rangle==_{\text {def }} \int_{\mathbb{R}^{n}} F \bar{G} d x$. Because of the Parseval identity we obtain also

$$
\begin{aligned}
I= & \left|\left\langle\hat{u}_{0},\left(1+|\xi|^{2}\right)^{(m-1+2 s) / 4} \int_{\mathbb{R}} e^{+i t p(\xi)} \mathscr{F}_{2}(\chi f) d t\right\rangle\right| \\
= & \left|\left\langle\hat{u}_{0},\left(1+|\xi|^{2}\right)^{(m-1+2 s) / 4} \mathscr{F}(\chi f)(-p(\xi) / 2 \pi, \xi)\right\rangle\right| \\
\leq & \left(\int_{\mathbb{R}^{n}}\left(1+|\xi|^{2}\right)^{s}\left|\hat{u}_{0}(\xi)\right|^{2} d \xi\right)^{1 / 2} \\
& \times\left(\int_{\mathbb{R}^{n}}\left(1+|\xi|^{2}\right)^{(m-1) / 2}|\mathscr{F}(\chi f)(-p(\xi) / 2 \pi, \xi)|^{2}\right)^{1 / 2}
\end{aligned}
$$

and by Theorem 1.1 with $q=2, \alpha=(m-1) / 2$ we obtain finally

$$
I \leq C_{\chi}\left\|u_{0}\right\|_{H^{s}\left(\mathbb{R}^{n}\right)}\|f\|_{L^{2}\left(\mathbb{R}^{n+1}\right)},
$$

which implies $(2.1)$ by duality and density.

Using Remark 1.2 instead of Theorem 1.1, we can obtain an $L^{q}, q \neq 2$, version of Theorem 2.1.

Theorem 2.2. We assume that $p$ satisfies (0.4), (0.5), and (0.6). Let $u_{0} \in$ $L^{q}\left(\mathbb{R}^{n}\right), 1 \leq q \leq 2$. Then for $\chi \in C_{0}^{\infty}\left(\mathbb{R}^{n+1}\right)$ of the form given in Theorem 1.1, the solution $u$ of $(0.1),(0.2)$ satisfies

$$
\left[\int_{\mathbb{R}^{n+1}} \chi^{r}(t, x)\left|(I-\Delta)^{\alpha / 2} u(t, x)\right|^{r} d x d t\right]^{1 / r} \leq C_{\chi}^{\prime}\left\|u_{0}\right\|_{L^{q}\left(\mathbb{R}^{n}\right)},
$$

where $C_{\chi}^{\prime}$ is the constant given by (1.16) and $\alpha, r, q$ satisfy

$$
\left\{\begin{array}{l}
r \geq 2, \\
\alpha<(m+n-1) / r-n / q .
\end{array}\right.
$$

In other words, $u \in L^{r}\left(-T, T ; H_{u ; \operatorname{loc}}^{\alpha, r}\left(\mathbb{R}^{n}\right)\right)$ for every $T>0$.

Proof. For $f \in \mathscr{S}\left(\mathbb{R}^{n+1}\right)$ we compute

$$
I=\left|\int_{\mathbb{R}^{n+1}} \chi(t, x)(I-\Delta)^{\alpha / 2} u(t, x) \overline{f(t, x)} d t d x\right| .
$$

As in the proof of Theorem 2.1, now using Hölder's inequality, we get

$$
\begin{gathered}
I \leq\left\|\hat{u}_{0}\right\|_{L^{q^{\prime}}}\left(\int_{\mathbb{R}^{n}}\left(1+|\xi|^{2}\right)^{\alpha q / 2}|\mathscr{F}(\chi f)(-p(\xi) / 2 \pi, \xi)|^{q} d \xi\right)^{1 / q}, \\
I \leq C\left\|u_{0}\right\|_{q}\left(\int_{\mathbb{R}^{n}}\left(1+|\xi|^{2}\right)^{\alpha q / 2}|\mathscr{F}(\chi f)(-p(\xi) / 2 \pi, \xi)|^{q} d \xi\right)^{1 / q}
\end{gathered}
$$

by the Hausdorff-Young inequality.

We can estimate the integral in (2.5) using (1.13) provided $\alpha$ satisfies (2.4) (which is exactly (1.12)) and we obtain

$$
I \leq C_{\chi}^{\prime}\left\|u_{0}\right\|_{q}\|f\|_{L^{r^{\prime}\left(\mathbb{R}^{n+1}\right)}},
$$

which gives (2.3) by an obvious duality and density argument. 
Corollary 2.1. The assumptions are those of Theorem 2.2. We assume moreover that $u_{0} \in H^{s, q}\left(\mathbb{R}^{n}\right), \quad 1 \leq q \leq 2, s \geq 0$. Then the corresponding solution $u$ of (0.1) satisfies

$$
u \in L^{r}\left(-T, T ; H_{u ; \text { loc }}^{s+\alpha, r}\left(\mathbb{R}^{n}\right)\right)
$$

for every $T>0$, where $r$ and $\alpha$ are given by (2.4).

As we mentioned in $\S 1$ (Remark 1.3), in the case $n=1$ we can derive a result which is global in time.

Proposition 2.1. We assume here that $n=1$ and that $p$ satisfies (0.4) with $R=0$ and $\left|p^{\prime}(\xi)\right| \geq C_{2}|\xi|^{m-1}$, for all $\xi \in \mathbb{R}$.

(i) Let $u_{0} \in H^{s}(\mathbb{R}), s \geq-(m-1) / 2$. Then the solution $u$ of $(0.1),(0.2)$ satisfies $u \in L^{2}\left(\mathbb{R} ; H_{u ; \text { loc }}^{s+(m-1) / 2}(R)\right)$. More precisely, for every $\chi \in \mathscr{S}(\mathbb{R})$ one has

$$
\|\chi u\|_{L^{2}\left(\mathbb{R}_{+} ; H^{s+(m-1) / 2(\mathbb{R}))}\right.} \leq C\left\|u_{0}\right\|_{H^{s}}\|\chi\|_{2} .
$$

(ii) Let $u_{0} \in \mathscr{S}(\mathbb{R})$ be such that $\left(I-d^{2} / d x^{2}\right)^{s / 2} u_{0} \in \mathscr{F}^{-1} L^{q}(\mathbb{R}), 1 \leq q \leq 2$, $s \geq-(m-1) / q^{\prime}, 1 / q+1 / q^{\prime}=1$. Then for every $\chi \in \mathscr{S}(\mathbb{R})$

$$
\chi\left(I-d^{2} / d x^{2}\right)^{s / 2+(m-1) / 2 q^{\prime}} u \in L^{q^{\prime}}\left(\mathbb{R} ; L_{u ; \operatorname{loc}}^{q^{\prime}}(\mathbb{R})\right) .
$$

Moreover,

$$
\left\|\chi\left(I+d^{2} / d x^{2}\right)^{s / 2+(m-1) / 2 q^{\prime}} u\right\|_{L^{q^{\prime}}\left(\mathbb{R}^{2}\right)} \leq C\left\|\left(1+\xi^{2}\right)^{s / 2} \hat{u}_{0}\right\|_{q}\left\{\|\chi\|_{q^{\prime}}+\|\hat{\chi}\|_{q}\right\} .
$$

Proof. let $a \in \mathscr{S}\left(\mathbb{R}^{2}\right)$ and $\chi \in \mathscr{S}(\mathbb{R})$.

(i) We evaluate

$$
I=\int a(t, x) \overline{\chi(x)} \overline{|D|^{(m-1) / 2+s} u(t, x)} d t d x,
$$

where $\widehat{|D| f}(\xi)=|\xi| \hat{f}(\xi)$. By the Parseval identity

$$
\begin{aligned}
I & =\int d t \int \mathscr{F}_{2}(\bar{\chi} a)(t, \xi) \mathscr{F}_{2} \overline{\left(|D|^{(m-1) / 2+s} u\right)(t, \xi)} d \xi \\
& =\int d t \int \mathscr{F}_{2}(\bar{\chi} a)(t, \xi) e^{i t p(\xi)}|\xi|^{(m-1) / 2+s} \overline{\hat{u}_{0}(\xi)} d \xi \\
& =\int \mathscr{F}(\bar{\chi} a)(-p(\xi) / 2 \pi, \xi)|\xi|^{(m-1) / 2+s} \hat{u}_{0}(\xi) d \xi
\end{aligned}
$$

Therefore

$$
|I| \leq\left\|u_{0}\right\|_{H^{s}}\left(\int|\mathscr{F}(\bar{\chi} a)(-p(\xi) / 2 \pi, \xi)|^{2}|\xi|^{m-1} d \xi\right)^{1 / 2} \leq C\left\|u_{0}\right\|_{H^{s}}\|\chi\|_{2}\|a\|_{2,2}
$$

which obviously proves (2.6).

(ii) We evaluate

$$
J=\int a(t, x) \overline{\chi(x)} \overline{\left(I-d^{2} / d x^{2}\right)^{s / 2+(m-1) / 2 q^{\prime}} u(t, x)} d t d x .
$$


Again, by the Parseval identity,

$$
J=\int d t \int \mathscr{F}_{2}(\bar{\chi} a)(t, \xi)\left(1+\xi^{2}\right)^{s / 2+(m-1) / 2 q^{\prime}} e^{i t p(\xi)} \overline{\hat{u}}_{0}(\xi) d \xi,
$$

and by the hypothesis on $u_{0}$,

$$
\begin{aligned}
&|J| \leq\left\|\left(1+\xi^{2}\right)^{s / 2} \hat{u}_{0}\right\|_{q}[\left.\int_{\mathbb{R}}|\mathscr{F}(\bar{\chi} a)(-p(\xi) / 2 \pi, \xi)|^{q^{\prime}}\left(1+\xi^{2}\right)^{(m-1) / 2} d \xi\right]^{1 / q^{\prime}} \\
& \leq C\left\|\left(1+\xi^{2}\right)^{s / 2} \hat{u}_{0}\right\|_{q} {\left[\left(\int_{\mathbb{R}}|\mathscr{F}(\bar{\chi} a)(-p(\xi) / 2 \pi, \xi)|^{q^{\prime}} d \xi\right)^{1 / q^{\prime}}\right.} \\
&\left.+\left(\int_{\mathbb{R}}|\mathscr{F}(\bar{\chi} a)(-p(\xi) / 2 \pi, \xi)|^{q^{\prime}}|\xi|^{m-1} d \xi\right)^{1 / q^{\prime}}\right] \\
& \leq C\left\|\left(1+\xi^{2}\right)^{s / 2} \hat{u}_{0}\right\|_{q}\left\{\|\hat{\chi}\|_{q}\left(\int_{\mathbb{R}^{2}}|\mathscr{F} a|^{q^{\prime}}(-p(\xi) / 2 \pi, \eta) d \eta d \xi\right)^{1 / q^{\prime}}\right. \\
&\left.+\left(\|\chi\|_{q^{\prime}}+\|\hat{\chi}\|_{q}\right)\|a\|_{q, q}\right\}
\end{aligned}
$$

where we have used the estimate of $\operatorname{Sup}_{\xi \in \mathbb{R}}|\mathscr{F}(\chi a)(\tau, \xi)|$ given in the proof of Remark 1.3 and (1.17) in the case $R=0$. Finally, we obtain with the Hausdorff-Young inequality

$$
|J| \leq C\left\|\left(1+\xi^{2}\right)^{s / 2} \hat{u}_{0}\right\|_{q}\left\{\|\chi\|_{q^{\prime}}+\|\hat{\chi}\|_{q}\right\}\|a\|_{q, q}
$$

which proves $(2.7)$.

Remark 2.2. Remark 2.1 and (2.5) imply that $u \in L^{2}\left(\mathbb{R}_{+} ; C_{b}^{k}(\mathbb{R})\right)$ provided $u_{0} \in H^{s}(\mathbb{R}), s>(2+2 k-m) / 2$ (this is the case for the linearlized $\mathrm{K}-\mathrm{dV}$ equation if $s>(2 k-1) / 2)$.

\section{LOCAL SMOOTHING PROPERTY FOR THE NONHOMOGENEOUS CASE}

In this section we derive local smoothing properties for the inhomogeneous equation

$$
\begin{gathered}
\partial u / \partial t+i P(D) u=F \quad \text { in } \mathbb{R} \times \mathbb{R}^{n}, \\
u(0, x)=u_{0}(x) \quad \text { in } \mathbb{R}^{n},
\end{gathered}
$$

where $P(D)$ is as in $\S 2$. As a consequence we shall obtain local smoothing results for Schrödinger equations with a large class of potentials and some results in the nonlinear case (cf. §5).

Theorem 3.1. We assume that $p$ satisfies (0.4), (0.5), and (0.6). Let $s \geq$ $-(m-1) / 2, u_{0} \in H^{s}\left(\mathbb{R}^{n}\right), F \in L_{\text {loc }}^{1}\left(\mathbb{R} ; H^{s, q}\left(\mathbb{R}^{n}\right)\right), 1 \leq q \leq 2$. Let $\alpha$ be given by (1.1). Then the solution $u$ of $(3.1)$, (3.2) belongs to $L^{2}\left(-T, T ; H_{u ; \operatorname{loc}}^{s+\alpha}\left(\mathbb{R}^{n}\right)\right)$ 
for every $T>0$. Moreover, for every $\chi \in C_{0}^{\infty}\left(\mathbb{R}^{n}\right)$ of the form given in Theorem 1.1 and which is supported in $[-T, T] \times \mathbb{R}^{n}$, one has

$$
\begin{aligned}
& \left(\int_{\mathbb{R}^{n}} \chi^{2}(t, x)\left|(I-\Delta)^{(s+\alpha) / 2} u(t, x)\right|^{2} d x d t\right)^{1 / 2} \\
& \quad \leq C_{\chi}\left(\left\|u_{0}\right\|_{H^{s}\left(\mathbb{R}^{n}\right)}+\|F\|_{L^{1}\left(-T, T ; H^{\left.s, q\left(\mathbb{R}^{n}\right)\right)}\right)}\right)
\end{aligned}
$$

where $C_{\chi}$ is as in Theorem 1.1 .

Proof. We use the Duhamel representation formula

$$
u(t, \cdot)=e^{-i t P(D)} u_{0}+\int_{0}^{t} e^{-i(t-\tau) P(D)} F(\tau, \cdot) d \tau .
$$

By Theorem 2.1, $e^{-i t P(D)} u_{0}$ lies in $L_{\text {loc }}^{2}\left(\mathbb{R}^{n} ; H_{u ; \text { loc }}^{s+(m-1) / 2}\left(\mathbb{R}^{n}\right)\right)$; therefore it is sufficient to consider the integral term in (3.4).

Let $f \in \mathscr{S}\left(\mathbb{R}^{n+1}\right)$ be supported in $[-T, T] \times \mathbb{R}^{n}$. We set

$$
I=\int_{\mathbb{R}^{n+1}} \chi(t, x)(I-\Delta)^{(s+\alpha) / 2} \int_{0}^{t} e^{-i(t-\tau) P(D)} F(\tau, x) d \tau \overline{f(t, x)} d t d x .
$$

Using the Parseval identity in the $x$-variables and interchanging the order of integration, we get

$$
\begin{aligned}
I & =\int_{-T}^{T} d t \int_{\mathbb{R}^{n}} \mathscr{F}_{2}(\chi \bar{f})(t, \xi)\left(1+|\xi|^{2}\right)^{(s+\alpha) / 2} \int_{0}^{t} e^{-i(t-\tau) p(\xi)} \mathscr{F}_{2} F(\tau, \xi) d \tau d \xi \\
& =\int_{-T}^{T} d \tau \int_{\mathbb{R}^{n}}\left(1+|\xi|^{2}\right)^{(s+\alpha) / 2} \mathscr{F}_{2} F(\tau, \xi)\left(\int_{\tau}^{T} e^{-i t p(\xi)} \mathscr{F}_{2}(\chi \bar{f})(t, \xi) d t\right) e^{i \tau p(\xi)} d \xi .
\end{aligned}
$$

For $\tau \in[-T, T]$ we define $f_{\tau}$ by

$$
\left(f_{\tau}\right)(t, x)=\chi_{[\tau, T]}(t) f(t, x)
$$

where $\chi_{[\tau, T]}$ is the characteristic function of $[\tau, T]$. Therefore,

$$
\int_{\tau}^{T} e^{-i t p(\xi)} \mathscr{F}_{2}(\chi \bar{f})(t, \xi) d t=\mathscr{F}\left(\chi \bar{f}_{\tau}\right)(p(\xi) / 2 \pi, \xi)
$$

and $I$ becomes

$$
I=\int_{-T}^{T} d \tau \int_{\mathbb{R}^{n}}\left(1+|\xi|^{2}\right)^{(s+\alpha) / 2} \mathscr{F}_{2} F(\tau, \xi) \mathscr{F}\left(\chi \bar{f}_{\tau}\right)(p(\xi) / 2 \pi, \xi) e^{i \tau p(\xi)} d \xi .
$$

Then

$$
\begin{aligned}
|I| \leq & \int_{-T}^{T}\left(\int_{\mathbb{R}^{n}}\left(1+|\xi|^{2}\right)^{s q^{\prime} / 2}\left|\mathscr{F}_{2} F(\tau, \xi)\right|^{q^{\prime}} d \xi\right)^{1 / q^{\prime}} \\
& \left.\quad \times\left(\int_{\mathbb{R}^{n}}\left(1+|\xi|^{2}\right)^{\alpha q / 2}\left|\mathscr{F}\left(\chi \bar{f}_{\tau}\right)(p(\xi) / 2 \pi, \xi)\right|^{q} d \xi\right)^{1 / q}\right\} d \tau \\
\leq & \quad \text { by Theorem } 1.1 \text { and the Hausdorff-Young Theorem) } \\
\leq & C_{\chi} \int_{-T}^{T}\|F(\tau, \cdot)\|_{H^{s . q\left(\mathbb{R}^{n}\right)}}\left\|f_{\tau}\right\|_{L^{2}\left(\mathbb{R}^{n+1}\right)} d \tau \\
\leq & C_{\chi}\|f\|_{L^{2}\left(\mathbb{R}^{n+1}\right)}\|F\|_{L^{1}\left(-T, T ; H^{\left.s . q\left(\mathbb{R}^{n}\right)\right)}\right.} .
\end{aligned}
$$


Proposition 3.1. Assume that $p$ satisfies (0.4), (0.5), and (0.6). Let $s \geq$ $-(m-1) / 2, u_{0} \in H^{s, q}\left(\mathbb{R}^{n}\right), 1 \leq q \leq 2, F \in L_{\text {loc }}^{1}\left(\mathbb{R} ; H^{s, q}\left(\mathbb{R}^{n}\right)\right)$. Then, for every $T>0$, the solution $u$ of (3.1), (3.2) belongs to $L^{r}\left(-T, T ; H_{u ; \text { loc }}^{s+\alpha}\left(\mathbb{R}^{n}\right)\right)$ provided $\alpha, r, q$ satisfy

$$
\left\{\begin{array}{l}
r \geq 2 \\
\alpha<(m+n-1) / r-n / q .
\end{array}\right.
$$

Proof. First, we use Corollary 2.1 to show that $e^{-i t P(D)} u_{0}$ belongs to $L^{r}\left(-T, T ; H_{u ; \text { loc }}^{s+r}\left(\mathbb{R}^{n}\right)\right.$ ) where $\alpha$ is given by (2.4) (which is (3.6)).

To deal with the integral term in (3.4) we apply (1.13) instead of Theorem 1.1 to majorize the second integral in (3.5).

As an application of Theorem 3.1, we consider Schrödinger equations with a real time dependent potential $V(t, x)$

$$
\begin{gathered}
i \partial u / \partial t+\Delta u+V(t, x) u=0, \quad \text { in } \mathbb{R} \times \mathbb{R}^{n}, \\
u(0, x)=u_{0}(x), \quad \text { in } \mathbb{R}^{n} .
\end{gathered}
$$

Corollary 3.1. Let $p>\operatorname{Max}(1, n / 2)$ and $\alpha, \beta$ real such that

$$
\frac{4 n p}{2 p(2 n+1)-n^{2}}<\alpha, \beta \leq \frac{2 p}{p-n / 2} .
$$

Assume that $V=V_{1}+V_{2}, \quad V_{1} \in L_{\mathrm{loc}}^{p}\left(\mathbb{R}, L^{\alpha}\left(\mathbb{R}^{n}\right)\right), \quad V_{2} \in L_{\mathrm{loc}}^{\infty}\left(\mathbb{R} ; L^{\beta}\left(\mathbb{R}^{n}\right)\right)$. Then the solution $u$ of (3.7), (3.8) corresponding to $u_{0} \in L^{2}\left(\mathbb{R}^{n}\right)$ satisfies $u \in$ $L^{2}\left(-T, T ; H_{\mathrm{loc}}^{s}\left(\mathbb{R}^{n}\right)\right)$ for every $T>0$, where $s>0$ is to be made explicit in the proof.

Proof. Under the assumptions of Corollary 3.1, a result of Yajima [19, Theorem 1.1] implies that $u \in L_{\text {loc }}^{2 p /(p-1)}\left(\mathbb{R} ; L^{4 p / n}\left(\mathbb{R}^{n}\right)\right)$. Hence

$$
V_{i} u \in L_{\mathrm{loc}}^{2 p /(p+1)}\left(\mathbb{R} ; L^{q_{i}}\left(\mathbb{R}^{n}\right)\right), \quad i=1,2,
$$

where $q_{1}=4 p \alpha /(n \alpha+4 p), q_{2}=4 p \beta /(4 p+n \beta)$. Our assumptions on $\alpha, \beta$ imply $1 \leq 2 n /(n+1)<q_{i} \leq 2, i=1,2$.

From Theorem 3.1 we can therefore infer that for every $T>0, u \in$ $L^{2}\left(-T, T ; H_{\mathrm{loc}}^{s}\left(\mathbb{R}^{n}\right)\right)$ where $s<\operatorname{Min}\left(1 / 2, d_{1}, d_{2}\right), d_{i}=1 / 2-\left(\left(2-q_{i}\right) / 2 q_{i}\right) n$ (note that $d_{i}>0$ for $i=1,2$ since $q_{i}>2 n /(n+1)$, and that one can take $s=1 / 2=d_{1}=d_{2}$ when $q_{1}=q_{2}=2$, which corresponds to the case $\alpha=\beta=2 p /(p-n / 2))$.

Remark 3.1. If $V \in L_{\text {loc }}^{\infty}\left(\mathbb{R} ; L^{\alpha}\left(\mathbb{R}^{n}\right)\right)$, in particular if $V$ is time independent and belongs to $L^{\alpha}\left(\mathbb{R}^{n}\right)$, Corollary 3.1 gives a local smoothing effect provided $2<\alpha \leq+\infty$. More precisely, the solution $u$ of (3.7), (3.8) with $u_{0} \in L^{2}\left(\mathbb{R}^{n}\right)$ belongs to $L^{2}\left(-T, T ; H_{\text {loc }}^{1 / 2}\left(\mathbb{R}^{n}\right)\right)$ for all $T>0$. In effect, if $\alpha$ is given in the range $2<\alpha<+\infty$, and if $p$ is defined by $\alpha=2 p /(p-n / 2)$, one can write

$$
V=\frac{1}{2} V+\frac{1}{2} V \in L_{\mathrm{loc}}^{p}\left(\mathbb{R} ; L^{\alpha}\left(\mathbb{R}^{n}\right)\right)+L_{\mathrm{loc}}^{\infty}\left(\mathbb{R} ; L^{\alpha}\left(\mathbb{R}^{n}\right)\right)
$$


and apply Corollary 3.1. If $\alpha=+\infty$, then $V u \in L_{\text {loc }}^{1}\left(\mathbb{R}_{+} ; L^{2}\left(\mathbb{R}^{n}\right)\right)$ and one can apply Theorem 3.1 directly.

4. LOCAL SMOOTHING EFFECTS FOR DISPERSIVE SYSTEMS

In this section we study linear dispersive systems of the following type

$$
\left\{\begin{array}{l}
\underline{u}_{t}+i \underline{\underline{P}}(D) \underline{u}=0, \quad \text { in } \mathbb{R} \times \mathbb{R}^{n}, \\
\underline{u}(0, \cdot)=\underline{u}_{0},
\end{array}\right.
$$

where $u=u(t, x): \mathbb{R} \times \mathbb{R}^{n} \rightarrow \mathbb{R}^{2}$ or $\mathbb{C}^{2}$, and $\underline{\underline{P}}(\xi)$ is a real matrix symbol

$$
\underline{\underline{P}}(\xi)=\left(\begin{array}{ll}
p_{1}(\xi) & p_{3}(\xi) \\
p_{4}(\xi) & p_{2}(\xi)
\end{array}\right), \quad \xi \in \mathbb{R}^{n} .
$$

The coefficients $p_{i}$ will satisfy

$$
\begin{array}{r}
p_{i} \in L_{\mathrm{loc}}^{\infty}\left(\mathbb{R}^{n}\right), \quad 1 \leq i \leq 4, \\
\frac{\left(p_{1}(\xi)-p_{2}(\xi)\right)^{2}}{4}+p_{3}(\xi) p_{4}(\xi)=b^{2}(\xi)>0
\end{array}
$$

for all $\xi \in \mathbb{R}^{n},|\xi| \geq M \geq 0$

(4.4) There exists $A^{2}>0$ such that

$$
\begin{aligned}
& \left(p_{3}(\xi)-p_{4}(\xi)\right)^{2} \leq A^{2}\left(\frac{\left(p_{1}(\xi)-p_{2}(\xi)\right)^{2}}{4}+p_{3}(\xi) p_{4}(\xi)\right) \\
& \quad \text { for all } \xi \in \mathbb{R}^{n},|\xi| \geq M \geq 0 .
\end{aligned}
$$

(4.5) There exists $R \geq M$ such that the functions

$$
\tau_{ \pm}(\xi)=\frac{p_{1}(\xi)+p_{2}(\xi)}{2} \pm b(\xi)
$$

are differentiable for $|\xi|>R$ and satisfy (0.5), (0.6) (with $p$ replaced by $\tau_{ \pm}$).

Systems like (4.1) are often encountered in the theory of dispersive long waves of small amplitude. Here are some examples.

Examples 4.1. (i) Linearized Boussinesq type systems (see [13])

$$
\left\{\begin{array}{l}
u_{t}+\alpha_{1} v_{x x x}+\alpha_{2} v_{x x x x x}=0, \\
v_{t}+\beta_{1} u_{x x x}+\beta_{2} u_{x x x x x}=0,
\end{array} \quad t \in \mathbb{R}, x \in \mathbb{R},\right.
$$

with $\alpha_{2} \beta_{2}>0$ (or $\alpha_{1} \beta_{1}>0$ if $\alpha_{2}=\beta_{2}=0$ ). This system is a bidirectional model for water waves.

(ii) Linearized systems for the interaction of long internal waves in two pycnoclines [12].

The pycnocline is the region between two fluids of different densities. It can support a variety of interesting waves. The following (linearized) system describes the interaction of long internal waves in two close pycnoclines (see [1] 
for a study of the Cauchy problem associated to the nonlinear system); here $u$ and $v$ are the amplitudes of the waves.

$$
\left\{\begin{array}{l}
u_{t}-\beta_{1} H_{1} u_{x}-\beta_{1} H_{2} v_{x}=0, \quad x \in \mathbb{R}, t \in \mathbb{R}, \\
v_{t}-\beta_{2} H_{3} v_{x}-\beta_{2} H_{2} u_{x}=0,
\end{array}\right.
$$

where

$$
\begin{aligned}
\hat{H}_{1}(\xi) & =\frac{1}{\delta_{1}} \xi \operatorname{coth} \frac{\xi}{\delta_{1}}+\frac{1}{\delta_{2}} \xi \operatorname{coth} \frac{\xi}{\delta_{2}} \\
\hat{H}_{3}(\xi) & =\frac{1}{\delta_{3}} \xi \operatorname{coth} \frac{\xi}{\delta_{3}}+\frac{1}{\delta_{2}} \xi \operatorname{coth} \frac{\xi}{\delta_{2}}
\end{aligned}
$$

while $H_{2}$ is a smoothing operator. $\delta_{1}, \delta_{2}, \delta_{3}$ are the depths of the fluid layers.

(iii) Strong interaction between internal solitary waves (cf. [6]). The strong interaction of weakly nonlinear large internal gravity waves occurs when the wave phase speeds are nearly equal, although the waves belong to different modes. It is described by a pair of coupled $\mathrm{K}-\mathrm{dV}$ equations for the amplitudes $u$ and $v$; the linearized system can be written

$$
\left\{\begin{array}{l}
u_{t}+u_{x x x}+a_{3} v_{x x x}=0, \\
b_{1} v_{t}+b_{2} a_{3} u_{x x x}+r v_{x}+v_{x x x}=0, \quad b_{2}>0 .
\end{array}\right.
$$

(iv) Boussinesq type equations. We consider the following scalar equation (where $P_{3}, P_{4}$ have real symbols)

$$
\begin{cases}u_{t t}+P_{3}(D) P_{4}(D) u=0, & x \in \mathbb{R}^{n}, t \in \mathbb{R} \\ u(0, \cdot)=u_{0}, & u_{t}(0, \cdot)=u_{1}\end{cases}
$$

which is clearly equivalent to the system of "Schrödinger" type

$$
\left\{\begin{array}{l}
u_{t}+i P_{3}(D) v=0 \\
v_{t}+i P_{4}(D) u=0 \\
u(0, \cdot)=u_{0}, \quad v(0, \cdot)=v_{0} \quad\left(=i P_{3}(D)^{-1} u_{1} \text { if } P_{3} \text { is invertible }\right) .
\end{array}\right.
$$

A special case is the (linearized) Boussinesq equation

$$
\begin{cases}u_{t}+\Delta^{2} u-\Delta u=0, & x \in \mathbb{R}^{n}, t \in \mathbb{R}, \\ u(0, \cdot)=u_{0}, & u_{t}(0, \cdot)=u_{1}\end{cases}
$$

which is equivalent to the system

$$
\left\{\begin{array}{l}
u_{t}+i(\Delta v-v)=0, \quad x \in \mathbb{R}^{n}, t \in \mathbb{R}, \\
v_{t}+i \Delta u=0, \\
u(0, \cdot)=u_{0}, \quad v(0, \cdot)=-i(-\Delta+I)^{-1} u_{1} .
\end{array}\right.
$$

Another typical example is the equation

$$
\left\{\begin{array}{l}
u_{t t}+(-1)^{k} \Delta^{k} u=0, \quad x \in \mathbb{R}^{n}, t \in \mathbb{R}^{n}, k \geq 2, \\
u(0, \cdot)=u_{0}, \quad u_{t}(0, \cdot)=u_{1} .
\end{array}\right.
$$


The system (4.10) will fulfill conditions (4.2)-(4.5) provided the symbols $p_{3}, p_{4}$ of $P_{3}, P_{4}$ satisfy

$$
\left\{\begin{array}{l}
p_{i} \in L_{\mathrm{loc}}^{\infty}\left(\mathbb{R}^{n}\right), \quad i=3,4 \\
p_{3}(\xi) p_{4}(\xi)>0, \quad \text { for }|\xi| \geq M \geq 0 \\
\text { There exists } A^{2}>0 \text { such that } \\
\quad p_{3}^{2}(\xi)+p_{4}^{2}(\xi) \leq A^{2} p_{3}(\xi) p_{4}(\xi) \quad \text { for }|\xi| \geq M \geq 0 \\
\text { There exists } R \geq M \text { such that } p_{3}, p_{4} \text { are differentiable } \\
\text { for }|\xi| \geq R \text { and } p_{3} p_{4} \text { satisfies }(0.5),(0.6) .
\end{array}\right.
$$

In the case of equation (4.13), (4.14) will be satisfied provided one sets $P_{3}=$ $P_{4}=\Delta^{l}$ if $k=2 l$ or $P_{3}=P_{4}=(-\Delta)^{1 / 2} \Delta^{l}$ if $k=2 l+1$.

Before stating the smoothing properties for (4.1), we want to compute more explicitly the matrix $e^{-i t P(\xi)}$. In order to do so, we decompose $P$ in a diagonal matrix and a traceless matrix:

$$
P(\xi)=\frac{\operatorname{Tr} P(\xi)}{2} I+\left(P(\xi)-\frac{\operatorname{Tr} P(\xi)}{2} I\right) .
$$

Let us denote by

$$
\begin{gathered}
a(\xi)=\frac{\operatorname{Tr} P(\xi)}{2}=\frac{1}{2}\left(p_{1}(\xi)+p_{2}(\xi)\right), \\
d(\xi)=\operatorname{det}(P(\xi)-a(\xi) I), \\
=-\left(\frac{\left(p_{1}(\xi)-p_{2}(\xi)\right)^{2}}{4}+p_{3}(\xi) p_{4}(\xi)\right) .
\end{gathered}
$$

Now, for any $2 \times 2$ traceless matrix $\tilde{Q}$, we have $\tilde{Q}^{2}=-(\operatorname{det} \tilde{Q}) I$.

Let us denote by

$$
b(\xi)=|d(\xi)|^{1 / 2}=\left|\frac{\left(p_{1}(\xi)-p_{2}(\xi)\right)^{2}}{4}+p_{3}(\xi) p_{4}(\xi)\right|^{1 / 2}
$$

and by

$$
Q(\xi)=(1 / b(\xi))(P(\xi)-a(\xi) I)
$$

Then

$$
Q^{2}(\xi)= \pm I
$$

More precisely,

$$
Q^{2}=\operatorname{sign}\left(\frac{\left(p_{1}-p_{2}\right)^{2}}{4}+p_{3} p_{4}\right) I .
$$

If $Q^{2}=I$, then $e^{i \lambda Q}=I+i \lambda Q-\left(\lambda^{2} / 2 !\right) I-i\left(\lambda^{3} / 3 !\right) Q+\cdots$. So $e^{i \lambda Q}=(\cos \lambda) I+$ $i(\sin \lambda) Q$. If $Q^{2}=-I$, then $e^{i \lambda Q}=(\cosh \lambda) I+i(\sinh \lambda) Q$. Summarizing,

$$
\begin{aligned}
e^{i t P(\xi)}= & e^{i a(\xi) t}(\cos (b(\xi) t) I+i \sin (b(\xi) t) Q(\xi)) \\
& \text { if }\left(p_{1}-p_{2}\right)^{2} / 4+p_{3} p_{4}>0 \text { (which is our assumption (4.3)), }
\end{aligned}
$$




$$
\begin{aligned}
& e^{i t P(\xi)}=e^{i a(\xi) t}(\cosh (b(\xi) t) I+i \sinh (b(\xi) t) Q(\xi)) \\
& \text { if }\left(p_{1}-p_{2}\right)^{2} / 4+p_{3} p_{4}<0,
\end{aligned}
$$

and

$$
e^{i t P(\xi)}=e^{i a(\xi) t}(I+i t(P(\xi)-a(\xi) I)) \quad \text { if }\left(p_{1}-p_{2}\right)^{2} / 4+p_{3} p_{4}=0 .
$$

We see that if $\left(p_{1}(\xi)-p_{2}(\xi)\right)^{2} / 4+p_{3}(\xi) p_{4}(\xi)<0$ for large $|\xi|$ then the Cauchy problem for (4.1) is ill posed in the sense of Hadamard; we avoid this situation by assuming (4.3).

Thus we obtain the following expression for $e^{i t P(\xi)}$ :

$$
e^{i t P(\xi)}=e^{i \tau_{+}(\xi) t}\left(\frac{I+Q(\xi)}{2}\right)+e^{i \tau_{-}(\xi) t}\left(\frac{I-Q(\xi)}{2}\right)
$$

where

and

$$
\begin{aligned}
\tau_{ \pm}(\xi) & =a(\xi) \pm b(\xi), \\
Q(\xi) & =\frac{1}{b(\xi)}\left(\begin{array}{ll}
\frac{p_{1}(\xi)-p_{2}(\xi)}{2} & p_{3}(\xi) \\
p_{4}(\xi) & \frac{p_{2}(\xi)-p_{1}(\xi)}{2}
\end{array}\right)
\end{aligned}
$$

$$
a(\xi)=\frac{p_{1}(\xi)+p_{2}(\xi)}{2}, \quad b(\xi)=\left[\frac{\left(p_{1}(\xi)-p_{2}(\xi)\right)^{2}}{4}+p_{3}(\xi) p_{4}(\xi)\right]^{1 / 2}
$$

We are now ready to state a smoothing property similar to Theorem 2.1 .

Theorem 4.1. We assume that $P(\xi)$ satisfies (4.2)- (4.5). Let $s \geq-(m-1) / 2$. Then for $\chi \in C_{0}^{\infty}\left(\mathbb{R}^{n+1}\right)$ of the form given in Theorem 1.1, the solution $u$ of (4.1) associated to $u_{0} \in H^{s}\left(\mathbb{R}^{n}\right)^{2}$ satisfies

$$
\left(\int_{\mathbb{R}^{n+1}} \chi^{2}(t, x)\left|(I-\Delta)^{(m-1+2 s) / 4} u(t, x)\right|^{2} d x d t\right)^{1 / 2} \leq C_{\chi}\left\|u_{0}\right\|_{H^{s}\left(\mathbb{R}^{n}\right)^{2}}
$$

where $C_{\chi}$ is the constant given by (2.6). In particular,

$$
u \in L^{2}\left(-T, T ; H_{u ; \text { loc }}^{s+(m-1) / 2}\left(\mathbb{R}^{n}\right)\right)^{2} \quad \text { for every } T>0 .
$$

Proof. We denote $x \cdot \bar{y}$ the scalar product in $\mathbb{C}^{2}$ (or $\mathbb{R}^{2}$ accordingly). For any $f \in \mathscr{S}\left(\mathbb{R}^{n+1}\right)^{2}$, we have

$$
\begin{aligned}
& \iint \chi(t, x)(I-\Delta)^{s / 2+(m-1) / 4} u(t, x) \cdot \overline{f(t, x)} d x d t \\
& \quad=\iint(I-\Delta)^{s / 2+(m-1) / 4} u(t, x) \cdot(\overline{\bar{\chi} f})(t, x) d x d t \\
& \quad \underset{\text { def }}{=} \int\left\langle(I-\Delta)^{s / 2+(m-1) / 4} u(t, \cdot), \bar{\chi} f\right\rangle d t \\
& \quad=\int\left\langle(I-\Delta)^{s / 2+(m-1) / 4} e^{-i t P(D)} u_{0}, \bar{\chi} f\right\rangle d t \\
& \quad=\left\langle(I-\Delta)^{s / 2} u_{0}, \int(I-\Delta)^{(m-1) / 4} e^{i t P^{*}(D)}(\bar{\chi} f) d t\right\rangle
\end{aligned}
$$


where $P^{*}(D)$ is the operator whose symbol is the transposed matrix

$$
P^{*}(\xi)=\left(\begin{array}{ll}
p_{1}(\xi) & p_{4}(\xi) \\
p_{3}(\xi) & p_{2}(\xi)
\end{array}\right)
$$

Therefore (see (4.23)),

$$
e^{i t P^{*}(\xi)}=e^{i \tau_{+}(\xi) t}\left(\frac{I+Q^{*}(\xi)}{2}\right)+e^{i \tau_{-}(\xi) t}\left(\frac{I-Q^{*}(\xi)}{2}\right)
$$

where $\tau_{ \pm}$are given in (4.5) and $Q^{*}$ is the transpose of $Q$. We have thus

$$
\begin{aligned}
& \iint \chi(t, x)(I-\Delta)^{s / 2+(m-1) / 4} u(t, x) \cdot \overline{f(t, x)} d t d x \\
& \quad=\left\langle(I-\Delta)^{s / 2} u_{0},(I-\Delta)^{(m-1) / 4} \int e^{i t P^{*}(D)}(\chi f) d t\right\rangle .
\end{aligned}
$$

Since we deal with an $L^{2}$ theory, we have to estimate

$$
\begin{gathered}
\left\|(I-\Delta)^{(m-1) / 4} \int e^{i t P^{*}(D)}(\chi f) d t\right\|_{L^{2}\left(\mathbb{R}^{n}\right)}=(\text { Parseval }) \\
=\left\|\left(1+|\xi|^{2}\right)^{(m-1) / 4} \int e^{i t P^{*}(\xi)} \mathscr{F}_{2}(\chi f)(t, \xi) d t\right\|_{L^{2}\left(\mathbb{R}^{n}\right)} .
\end{gathered}
$$

Now, using (4.26)

$$
\begin{aligned}
& \int e^{i t P^{*}(\xi)} \mathscr{F}_{2}(\chi f)(t, \xi) d t \\
& \quad=\frac{I+Q^{*}(\xi)}{2} \mathscr{F}(\chi f)\left(-\frac{\tau_{+}(\xi)}{2 \pi}, \xi\right)+\frac{I-Q^{*}}{2} \mathscr{F}(\chi f)\left(-\frac{\tau_{-}(\xi)}{2 \pi}, \xi\right) .
\end{aligned}
$$

We have to estimate therefore

$$
\begin{aligned}
\int_{\mathbb{R}^{n}}\left(1+|\xi|^{2}\right)^{(m-1) / 2} \mid \frac{I+Q^{*}(\xi)}{2} \mathscr{F}( & \chi f)\left(-\frac{\tau_{+}(\xi)}{2 \pi}, \xi\right) \\
& +\left.\frac{I-Q^{*}(\xi)}{2} \mathscr{F}(\chi f)\left(-\frac{\tau_{-}(\xi)}{2 \pi}, \xi\right)\right|^{2} d \xi .
\end{aligned}
$$

This will be estimated if

$$
\int_{\mathbb{R}^{n}}\left(1+|\xi|^{2}\right)^{(m-1) / 2}\left|\frac{I \pm Q^{*}(\xi)}{2} \mathscr{F}(\chi f)\left(-\frac{\tau_{ \pm}(\xi)}{2 \pi}, \xi\right)\right|^{2} d \xi
$$

are both estimated. We recall that by (4.4) one has

$$
\left|\left(p_{3}-p_{4}\right)(\xi)\right|^{2} \leq A^{2}\left(\left(p_{1}-p_{2}\right)^{2} / 4+p_{3} p_{4}\right)(\xi) \text { for all }|\xi| \geq M .
$$


This implies that the norm of the $2 \times 2$ matrix $Q^{*}(\xi)$ is bounded uniformly in $\xi$, for $|\xi| \geq M$. Indeed,

$$
\begin{aligned}
\left\|Q^{*}(\xi)\right\|^{2} & \leq \operatorname{Tr} Q(\xi) Q^{*}(\xi)=\frac{1}{b^{2}}\left[\left(p_{1}-p_{2}\right)^{2} / 2+p_{3}^{2}+p_{4}^{2}\right](\xi) \\
& =\frac{1}{b^{2}}\left[2\left(\left(p_{1}-p_{2}\right)^{2} / 4+p_{3} p_{4}\right)+\left(p_{3}-p_{4}\right)^{2}\right](\xi) \\
& =2+\left(\left(p_{3}-p_{4}\right)^{2} / b^{2}\right)(\xi) \\
& \leq 2+A^{2} \text { for }|\xi| \geq M .
\end{aligned}
$$

Therefore (4.30) will be estimated provided we can bound

$$
\int_{\mathbb{R}^{n}}\left(1+|\xi|^{2}\right)^{(m-1) / 2}\left|\mathscr{F}(\chi f)\left(-\tau_{ \pm}(\xi) / 2 \pi, \xi\right)\right|^{2} d \xi
$$

Since by (4.5), $\tau_{ \pm}$satisfy $(0.4)-(0.6)$ we can apply Theorem 1.1. The remainder of the proof is as in Theorem 2.1 and we get

$$
\begin{aligned}
& \left|\iint \chi(t, x)(I-\Delta)^{s / 2+(m-1) / 4} u(t, x) \cdot \overline{f(t, x)} d x d t\right| \\
& \quad \leq C_{\chi}\left\|u_{0}\right\|_{H^{s\left(\mathbb{R}^{n}\right)}}\|f\|_{L^{2}\left(\mathbb{R}^{n+1)}\right)}, \quad f \in L^{2}\left(\mathbb{R}^{n+1}\right)
\end{aligned}
$$

which yields (4.25).

Examples 4.2. (i) In the case of (4.11), we obtain a solution $u$ in $L^{2}\left(-T, T ; H_{\text {loc }}^{1 / 2}\left(\mathbb{R}^{n}\right)\right), T>0$, provided $\left(u_{0}, u_{1}\right) \in L^{2}\left(\mathbb{R}^{n}\right) \times H^{-2}\left(\mathbb{R}^{n}\right)$.

(ii) In the case of (4.13) with $k=2$, let $\left(u_{0}, u_{1}\right) \in L^{2}\left(\mathbb{R}^{n}\right) \times L^{2 n /(n+2)}\left(\mathbb{R}^{n}\right)$, $n \geq 3$. Then the solution $u$ of (4.13) belongs to $L^{2}\left(-T, T ; H_{\mathrm{loc}}^{1 / 2}\left(\mathbb{R}^{n}\right)\right)$, for $T>0$. (Use the fact that $(-\Delta)^{-1} u_{1} \in L^{2}\left(\mathbb{R}^{n}\right)$ by the Hardy-Littlewood Sobolev theorem (cf. [16, p. 119]).

Remark 4.1. Condition (4.4) can be omitted in Theorem 4.1 if $p_{1}(\xi)+p_{2}(\xi)=$ 0 , so that $\tau_{+}(\xi)=-\tau_{-}(\xi)$, and if $u(-t, x)=u(t, x)$, for all $t \in \mathbb{R}$ and $x \in \mathbb{R}^{n}$. Indeed, going back to (4.29), (4.30), it follows that if $\mathscr{F}(\chi f)(-\tau, \xi)=$ $\mathscr{F}(\chi f)(\tau, \xi)$ then

$$
\begin{aligned}
\int e^{i t P^{*}(\xi)} \mathscr{F}_{2}(\chi f)(t, \xi) d t & =\mathscr{F}(\chi f)\left(-\tau_{+}(\xi) / 2 \pi, \xi\right)+Q^{*}(\xi)(0) \\
& =\mathscr{F}(\chi f)\left(-\tau_{+}(\xi) / 2 \pi, \xi\right) .
\end{aligned}
$$

This means that we can estimate the even part of $u(t, x)$; i.e.,

$$
\chi(I-\Delta)^{s / 2+(m-1) / 4}(u(t, \cdot)+u(-t, \cdot) / 2)
$$

without (4.4) if $p_{1}+p_{2} \equiv 0$.

Remark 4.2. In the examples of the type

$$
P(\xi)=\left(\begin{array}{ll}
p_{1}(\xi) & p_{3}(\xi) \\
\alpha p_{3}(\xi) & p_{2}(\xi)
\end{array}\right)
$$


condition (4.3) means $\alpha>0$ (and $\alpha<0$ would imply ill-posedness of the Cauchy problem). Condition (4.4) is automatically satisfied with $A=(\alpha+1) / \alpha$. Condition (4.5) is a growth condition at infinity and applies when $p_{3}$ dominates $p_{1}-p_{2}$ or when $p_{1}-p_{2}$ dominantes $p_{3}$.

Remark 4.3. One has, obviously, the analogues of Theorem 2.2, Corollary 2.1, and Proposition 2.1 for systems of the type (4.1). The same is true for the results of $\S 3$.

\section{LOCAL SMOOTHING: SOME NONLINEAR EXAMPLES}

We give two examples of nonlinear dispersive equations. The first one concerns the Schrödinger equation, the second a class of Korteweg-de Vries type equations.

We consider the Cauchy problem for the nonlinear Schrödinger equation (we restrict to spatial dimension $\leq 3$ for technical reasons)

$$
\left\{\begin{array}{l}
i \partial u / \partial t+\Delta u=F(u), \quad x \in \mathbb{R}^{n}, n \leq 3, \\
u(0, x)=u_{0}(x)
\end{array}\right.
$$

The assumptions on the function $F$ will be similar to those of Kato [11].

$$
\left.F \in C^{2}(\mathbb{C}, \mathbb{C}) ; \quad F(0)=0, \quad \mid F^{\prime}(z)\right)\left.|\leq M| z\right|^{p-1} \text { for }|z| \geq 1
$$

where

$$
1<p<5 \text { if } n=3 ; \quad 1<p<\infty \text { if } n \leq 2 .
$$

Under these assumptions, Kato [11] has proven the existence of a unique local solution $u \in C\left(-T, T ; H^{2}\left(\mathbb{R}^{n}\right)\right)$ provided $u_{0} \in H^{2}\left(\mathbb{R}^{n}\right)$. The next result gives a smoothness property of $u$.

Theorem 5.1. Under the assumptions (5.2), (5.3), let $u_{0} \in H^{2}\left(\mathbb{R}^{n}\right)$. Then there exists $a T>0$ and a unique solution $u$ of $(5.1)$, with $u \in C\left([-T, T] ; H^{2}\left(\mathbb{R}^{n}\right)\right) \cap$ $L^{2}\left(-T, T ; H_{u ; \text { loc }}^{5 / 2}\left(\mathbb{R}^{n}\right)\right)$.

Proof. The existence in $C\left([-T, T] ; H^{2}\left(\mathbb{R}^{n}\right)\right)$ for some $T>0$ and the uniqueness resuit from [11]. Concerning the smoothing property, it is sufficient to remark that $F(u) \in L^{\infty}\left([-T, T] ; H^{2}\left(\mathbb{R}^{n}\right)\right)$ and to use Theorem 3.1.

Remark 5.1. As noticed by Kato [11], the solution $u$ exists for all time ( $T=$ $+\infty)$, under the assumption that $F(z)=\partial H(z) / \partial \bar{z}$ where $H(z)$ is a realvalued function such that $H(0)=0$ and $H(z) \geq c|z|^{2}, c>0$.

Our second application to nonlinear problems will concern a class of nonlocal Korteweg-de Vries equations. This example is more involved since the nonlinearity involves an $x$-derivative. We consider

$$
\left\{\begin{array}{l}
\partial u / \partial t+u \partial u / \partial x+L \partial u / \partial x=0, \quad t \in \mathbb{R}, x \in \mathbb{R} \\
u(0, x)=u_{0}(x)
\end{array}\right.
$$


Here $L$ is defined by $\widehat{L v}(\xi)=q(\xi) v(\xi)$ and we set $P(D)=(\partial / \partial x) L$ to be consistent with the general notations of this paper. Therefore we set $p(\xi)=$ $\xi q(\xi)$ and assume that $p$ satisfies $(0.4),(0.5)$, and $(0.6)$.

It is well known (see for instance [14]) that if $u_{0}$ is smooth, say $u \in$ $H^{\infty}(\mathbb{R}) \equiv \bigcap_{s>0} H^{s}(\mathbb{R}),(5.4)$ possesses a unique local smooth solution $u \in$ $C^{\infty}\left(-T_{*}, T_{*} ; H^{\infty}(\mathbb{R})\right)$, where $T_{*}=T_{*}\left(\left\|u_{0}\right\|_{H^{2}}\right)$.

Our purpose is now to derive some a priori estimates on $u$ which will depend only on $\left\|u_{0}\right\|_{L^{2}}$ and which could be used to derive a local existence result for (5.4) with $L^{2}(\mathbb{R})$ initial data.

Obviously,

$$
\|u(t, \cdot)\|_{L^{2}(\mathbb{R})}=\left\|u_{0}\right\|_{L^{2}(\mathbb{R})} .
$$

Theorem 5.2. We assume $m>2$. Let $0<\alpha<\operatorname{Min}(m-2,(m-1) / 2)$, $\chi \in C_{0}^{\infty}(\mathbb{R}), T>0$. Then the solution $u$ of (5.4) satisfies

$$
\left\|\mathscr{F}\left(\chi \chi_{[-T, T]}|D|^{\alpha} u\right)\right\|_{L^{\infty}\left(\mathbb{R}^{2}\right)} \leq C(T ; \chi, \alpha)\left(\left\|u_{0}\right\|_{2}+\left\|u_{0}\right\|_{2}^{2}\right)
$$

where $c(T, \chi, \alpha) \leq c(\alpha, \chi)\left(1+T^{2}\right)$ and $|D|$ is the operator having for symbol $|\xi|$.

Proof. We use the Duhamel representation formula

$$
u(t, \cdot)=e^{-i t P(D)} u_{0}-\int_{0}^{t} e^{-i(t-s) P(D)} u u_{x}(s, \cdot) d s .
$$

We consider the second term in (5.7). Let $v(t, x)=\int_{0}^{t} e^{-i(t-s) P(D)} u u_{x}(s, \cdot) d s$. Let $a \in \mathscr{S}\left(\mathbb{R}^{2}\right)$ of the form $a=\psi * b, \psi, b \in \mathscr{S}\left(\mathbb{R}^{2}\right)$; the convolution is in $\mathbb{R}^{2}$. Then

$$
\begin{aligned}
& \left|\iint a(t, x) \chi(x)\left(|D|^{\alpha} v\right)(t, x) d t d x\right| \\
& \quad=\left|\int_{-T}^{T} d t \int_{\mathbb{R}}\left(\mathscr{F}_{2}(\chi a)\right)(t, \xi) \mathscr{F}\left(|D|^{\alpha} v\right)(t, \xi) d \xi\right| \\
& \quad \leq \pi \int_{-T}^{T} d s \int_{\mathbb{R}}|\xi|^{\alpha+1}\left|\left(u^{2}(s)\right)^{\wedge}(\xi)\right|\left|\mathscr{F}\left(\chi a_{s}\right)(p(\xi) / 2 \pi, \xi)\right| d \xi
\end{aligned}
$$

where $a_{s}(t)=\chi_{[s, T]}(t) a(t, x)$ (see the proof of Theorem 3.1 for a similar computation).

We can write $\chi(x)=\chi(x) \tilde{\chi}(x)$ with $\tilde{\chi} \equiv 1$ on $\operatorname{supp} \chi, \tilde{\chi} \in C_{0}^{\infty}(\mathbb{R})$ and thus

$$
\chi a_{s}=\chi \tilde{\chi} a_{s}=\chi\left(\tilde{\chi} \chi_{[s, T]}\right) a=\chi\left[\chi_{1}(\psi * b)\right]
$$

where $\chi_{1}(t, x)=\tilde{\chi}(x) \chi_{[s, T]}(t)$.

Now unfortunately $\mathscr{F} \chi_{1}=(\tilde{\chi})^{\wedge}(\xi) \hat{\chi}_{[s, T]}(\tau)$ is not in $L^{1}\left(\mathbb{R}^{2}\right)$ since

$$
\hat{\chi}_{[s, T]}(\tau)=\left(i e^{-2 i \pi \tau s} / 2 \pi \tau\right)\left[e^{-2 i \pi(T-s) \tau}-1\right],
$$

but for any $q>1,\left\|\mathscr{F} \chi_{1}\right\|_{L^{q}\left(\mathbb{R}^{2}\right)}<+\infty$. 
Since we assumed $0<\alpha<m-2$, we can find $q>1$ such that $m-1>$ $q(\alpha+1)+q-1($ take $q>1$ near 1$)$. Then

$$
\left\{\begin{array}{l}
\beta \underset{\text { def }}{=}(m-1) / q-(\alpha+1)>0 \quad \text { and } \\
\beta q^{\prime}>1 \quad\left(1 / q+1 / q^{\prime}=1\right)
\end{array}\right.
$$

so that

$$
\int_{|\xi| \geq 1}|\xi|^{-q^{\prime} \beta} d \xi<+\infty
$$

We estimate the integral

$$
\int_{\mathbb{R}}|\xi|^{\alpha+1}\left|u^{2}(s)^{\wedge}(\xi)\right|\left|\mathscr{F}\left(\chi a_{s}\right)(p(\xi) / 2 \pi, \xi)\right| d \xi \leq \int_{|\xi| \leq M}+\int_{|\xi| \geq M} .
$$

Obviously, by the conservation of the $L^{2}$ norm,

$$
\left|\int_{|\xi| \leq M}\right| \leq C\left\|u_{0}\right\|_{2}^{2}\left\|\chi a_{s}\right\|_{1} \leq C\left\|u_{0}\right\|_{2}^{2}\left\|\chi \chi_{1}\right\|_{1}\|\psi\|_{2}\|b\|_{2} .
$$

On the other hand, by (5.8), Hölder's inequality and the conservation of the $L^{2}$ norm

$$
\left|\int_{|\xi| \geq M}\right| \leq C\left(\int_{|\xi| \geq M}|\xi|^{m-1}\left|\mathscr{F}\left(\chi a_{s}\right)(p(\xi) / 2 \pi, \xi)\right|^{q} d \xi\right)^{1 / q}\left\|u_{0}\right\|_{2}^{2} .
$$

The last integral in (5.9) is estimated by the

Lemma 5.1. Let $1<q<+\infty, \chi \in C_{0}^{\infty}(\mathbb{R}) ; a=\chi_{1} \cdot(\psi * b)$ where $\chi_{1} \in \mathscr{S}\left(\mathbb{R}^{n}\right)$ has compact support in $t, \psi, b \in \mathscr{S}\left(\mathbb{R}^{2}\right)$. Then

$$
\begin{aligned}
& \left(\int_{\mathbb{R}^{n}}|\xi|^{m-1}|\mathscr{F}(\chi a)|^{q}(p(\xi), \xi), d \xi\right)^{1 / q} \\
& \quad \leq C\left(\left\|\chi \chi_{1}\right\|_{L^{1}\left(\mathbb{R}^{2}\right)}+\left\|\mathscr{F} \chi_{1}\right\|_{L^{q}\left(\mathbb{R}^{2}\right)}\right)\|b\|_{L^{2}\left(\mathbb{R}^{2}\right)}\|\psi\|_{L^{2}\left(\mathbb{R}^{2}\right)}
\end{aligned}
$$

where $C>0$ is an absolute constant.

Proof. One has (see the proof of (1.17) in Remark 1.3)

$$
\left(\int_{\mathbb{R}^{n}}|\xi|^{m-1}|\mathscr{F}(\chi a)|^{q}(p(\xi), \xi) d \xi\right)^{1 / q} \leq C\left(\|\chi a\|_{L^{1}\left(\mathbb{R}^{2}\right)}+\|\hat{\chi}\|_{L^{q^{\prime}(\mathbb{R})}}\|\mathscr{F} a\|_{L^{q}\left(\mathbb{R}^{2}\right)}\right) .
$$

Therefore it suffices to notice that

$$
\begin{aligned}
\|\chi a\|_{L^{1}\left(\mathbb{R}^{2}\right)} & \leq\left\|\chi \chi_{1}\right\|_{L^{1}\left(\mathbb{R}^{2}\right)}\|\psi * b\|_{L^{\infty}\left(\mathbb{R}^{2}\right)} \\
& \leq\left\|\chi \chi_{1}\right\|_{1}\|\psi\|_{2}\|b\|_{2}
\end{aligned}
$$

and by the convolution inequality

$$
\begin{aligned}
\|a\|_{L^{q}\left(\mathbb{R}^{2}\right)} & =\left\|\mathscr{F}\left(\chi_{1}\right) * \mathscr{F}(\chi * b)\right\|_{q} \\
& \leq\left\|\mathscr{F}\left(\chi_{1}\right)\right\|_{q}\|\mathscr{F}(\psi * b)\|_{1} \leq\left\|\mathscr{F}\left(\chi_{1}\right)\right\|_{q}\|\psi\|_{2}\|b\|_{2} .
\end{aligned}
$$


Using Lemma 5.1, (5.9), and (5.10), we obtain

$$
\left.\left|\int_{-T}^{T} \int_{\mathbb{R}}(\psi * b)(t, x) \chi(x)\right| D\right|^{\alpha} v(t, x) d t d x \mid \leq C(T ; \chi ; \alpha)\left\|u_{0}\right\|_{2}^{2}\|\psi\|_{2}\|b\|_{2}
$$

for all $\psi, b \in \mathscr{S}\left(\mathbb{R}^{2}\right)$, where $C(T ; \chi ; \alpha) \leq C(\chi, \alpha)\left(1+T^{2}\right)$. Let $w=$ $\chi(x) \chi_{[-T, T]}(t)|D|^{\alpha} v(t, x)$. (Recall that $\left.v(t, x)=\int_{0}^{t} e^{-i(t-s) P(D)} u u_{x}(s, \cdot) d s\right)$. Thus $(5.12)$ can be read as

$$
\left|\iint_{\mathbb{R}^{2}}(\psi * b) w d t d x\right| \leq\left\|u_{0}\right\|_{2}^{2}\|\psi\|_{2}\|b\|_{2} \text { for all } \psi, b \in \mathscr{S}\left(\mathbb{R}^{2}\right) .
$$

Therefore (use Parseval identity),

$$
\|\psi * w\|_{L^{2}\left(\mathbb{R}^{2}\right)} \leq C\left\|u_{0}\right\|_{2}^{2}\|\psi\|_{2} \text { for all } \psi \in \mathscr{S}\left(\mathbb{R}^{2}\right)
$$

or

$$
\|\mathscr{F} \psi \mathscr{F} w\|_{L^{2}\left(\mathbb{R}^{2}\right)} \leq c\left\|u_{0}\right\|_{2}^{2}\|\mathscr{F} \psi\|_{2} \text { for all } \psi \in \mathscr{S}\left(\mathbb{R}^{2}\right)
$$

which implies that $\mathscr{F} w \in L^{\infty}\left(\mathbb{R}^{2}\right)$ and satisfies

$$
|\mathscr{F}(w)(\tau, \xi)| \leq C\left\|u_{0}\right\|_{2}^{2} \text { a.e. in } \mathbb{R}^{2} .
$$

It remains to deal with the "linear" term $w_{0} \underset{\text { def }}{=} e^{-i t P(D)} u_{0}$ in (5.6). Now, if $\alpha \leq(m-1) / 2$, we already know (see $\S 3)$ that

$$
\left\|\chi_{[-T, T]} \chi(x)|D|^{\alpha} w_{0}\right\|_{L^{2}\left(\mathbb{R}^{2}\right)} \leq C(T ; \chi)\left\|u_{0}\right\|
$$

Therefore, since

$$
\chi_{[-T, T]} \chi(x)|D|^{\alpha} w_{0}=\left(\chi_{[-T, T]} \tilde{\chi}(x)\right)\left(\chi_{[-T, T]} \chi(x)|D|^{\alpha} w_{0}\right)
$$

belongs to $L^{1}\left(\mathbb{R}^{2}\right)$, its Fourier transform belongs to $L^{\infty}\left(\mathbb{R}^{2}\right)$. This together with (5.12) proves (5.5) and Theorem 5.2.

Corollary 5.1. The assumptions are those of Theorem 5.2. Consider the localized

$$
u_{l}(t, x)=\chi_{[-T, T]}(t) \chi(x) u(t, x) .
$$

Then there exists a constant $C$ depending on $\chi, \alpha, T$ and the norm $\left\|u_{0}\right\|_{2}$ only, such that

$$
\left|\mathscr{F}\left(u_{l}\right)(\tau, \xi)\right| \leq C(1+|\xi|)^{-\alpha}, \text { a.e. }(\tau, \xi) \in \mathbb{R}^{2} .
$$

Proof. By Theorem 5.2, we know that the function $\chi(x) \chi_{[-T, T]}|D|^{\alpha} u(t, x)$ has its Fourier transform in $L^{\infty}\left(\mathbb{R}^{2}\right)$. Let us write $u_{T}(t, x)=\chi_{[-T, T]}(t) u(t, x)$, so that

$$
u_{l}(t, x)=\chi(x) u_{T}(t, x)=\chi(x) \chi_{[-T, T]}(t) u_{T}(t, x)=\chi_{1}(t, x) u_{T}(t, x) .
$$


We have therefore

$$
\begin{gathered}
\mathscr{F}\left(|D|^{\alpha} u_{l}\right)(\tau, \xi)=|\xi|^{\alpha} \iint_{\mathbb{R}^{2}}\left(\mathscr{F} \chi_{1}\right)(\sigma, \eta)\left(\mathscr{F} u_{T}\right)(\tau-\sigma, \xi-\eta) d \sigma d \eta, \\
\mathscr{F}\left(\chi \chi_{[-T, T]}|D|^{\alpha} u\right)(\tau, \xi)=\iint_{\mathbb{R}^{2}} \mathscr{F}\left(\chi_{1}\right)(\sigma, \eta)|\xi-\eta|^{\alpha}\left(\mathscr{F} u_{T}\right)(\tau-\sigma, \xi-\eta) d \sigma d \eta
\end{gathered}
$$

(we used $\chi \chi_{[-T, T]}|D|^{\alpha} u=\chi_{1}|D|^{\alpha} u_{T}$ ). Subtracting, we get

$$
\begin{aligned}
& \left|\left\{\mathscr{F}\left(|D|^{\alpha} u_{l}\right)-\mathscr{F}\left(\chi \chi_{[-T, T]}|D|^{\alpha} u\right)\right\}(\tau, \xi)\right| \\
& \quad \leq\left.\iint_{\mathbb{R}^{n}}|| \xi\right|^{\alpha}-|\xi-\eta|^{\alpha}|| \mathscr{F} \chi_{1}(\sigma, \eta)|| \mathscr{F} u_{T}(\tau-\sigma, \xi-\eta) \mid d \sigma d \eta .
\end{aligned}
$$

Let us first assume $0 \leq \alpha \leq 1$. Then $\left.|| \xi\right|^{\alpha}-\left.|\xi-\eta|^{\alpha}\left|\leq C_{\alpha}\right| \eta\right|^{\alpha}$ and the right-hand side of (5.15) is majorized by

$$
C_{\alpha}\left\||D|^{\alpha} \chi_{1}\right\|_{L^{2}\left(\mathbb{R}^{2}\right)}\left\|u_{T}\right\|_{L^{2}\left(\mathbb{R}^{2}\right)} .
$$

Since $|D|^{\alpha} \chi_{1}=\chi_{[-T, T]}|D|^{\alpha} \chi$ we conclude that

$$
|\xi|^{\alpha}\left|\mathscr{F}\left(u_{l}\right)(\tau, \xi)\right| \leq c_{1}+c_{2} T\left\|u_{0}\right\|_{2} .
$$

Now, $\left|\mathscr{F}\left(u_{l}\right)(\tau, \xi)\right| \leq\left\|u_{l}\right\|_{1} \leq C T\left\|u_{0}\right\|_{2}$, and the corollary is proved in the case $\alpha \leq 1$. If $\alpha \geq 1$, one uses an obvious induction argument.

As an immediate consequence of Corollary 5.1, we obtain an a priori estimate in a Sobolev space.

Corollary 5.2. The assumptions are those of Theorem 5.2. Let

$$
\alpha=\operatorname{Min}(m-2,(m-1) / 2)
$$

and assume $\alpha>1 / 2$; then for every $a \in L^{2}(-T, T), \chi \in C_{0}^{\infty}(\mathbb{R})$, the function $u_{a}=\int_{-T}^{T} a(t-s) u(s, x) \chi(x) d s$ belongs to $L^{2}\left(-T, T ; H^{\delta}(\mathbb{R})\right)$ for any $\delta>0$ such that $\alpha>1 / 2+\delta$. Moreover,

$$
\left\|u_{a}\right\|_{L^{2}\left(-T, T ; H^{\delta}(\mathbb{R})\right)} \leq C\|a\|_{L^{2}(-T, T)},
$$

where

$$
C=C(\alpha, \chi)\left(1+T^{2}\right)\left(\left\|u_{0}\right\|_{2}+\left\|u_{0}\right\|_{2}^{2}\right)
$$

Proof. We have

$$
\mathscr{F}\left(u_{a}\right)(\tau, \xi)=\hat{a}_{T}(\tau) \mathscr{F}\left(u_{l}\right)(\tau, \xi) \quad \text { where } a_{T}=\chi_{[-T, T]} a .
$$

Thus

$$
\left.\left.|| \xi\right|^{\delta} \mathscr{F}\left(u_{a}\right)(\tau, \xi)|\leq C| \xi\right|^{\delta}|\hat{a}(\tau)|\left((1+|\xi|)^{2}\right)^{-\alpha / 2}=h(\tau, \xi) .
$$

Since $h \in L^{2}\left(\mathbb{R}^{2}\right)$ provided $\alpha>1 / 2+\delta$, we obtain (5.16).

\section{REFERENCES}

1. L. Abdelouhab, J. Bona, M. Felland, and J. C. Saut, Nonlocal models for nonlinear dispersive waves, Physica D, 1987.

2. M. Balabane, On a regularizing effect of Schrödinger type groups, Prè-publications mathématiques, Université Paris-Nord, no. 68, 1986. 
3. M. Balabane and H. A. Emami-rad, $L^{p}$ estimates for Schrödinger evolution equations, Trans. Amer. Math. Soc. 292 (1985), 357-373.

4. T. Cazenave and F. Weissler, The Cauchy problem for the nonlinear Schrödinger equation in $H^{1}$, preprint.

5. P. Constantin and J. C. Saut, Effets régularisants locaux pour des équations dispersives générales, C.R. Acad. Sci. Paris, Sér. I, 304 (1987), 407-410.

6. J. A. Gear and R. Grimshaw, Weak and strong interactions between internal solitary waves, Stud. Appl. Math., Elsevier, New York, LXX (1984), 235-258.

7. J. Ginibre and G. Velo, The global Cauchy problem for the nonlinear Schrödinger equation revisited, Ann. Inst. H. Poincaré. Anal. Non Linéaire, Vol. 2, no. 4, 1985, pp. 309-327.

8. N. Hayashi, K. Nakamitsu, and M. Tsutsumi, On solutions of the initial value problem for the nonlinear Schrödinger equation, J. Funct. Anal. 71 (1987), 218-245.

9. __ On solutions of the initial value problem for the nonlinear Schrödinger equation in one space dimension, Math. Z. 192 (1986), 637-650.

10. T. Kato, On the Cauchy problem for the (generalized) Korteweg-de Vries equation, Stud. Appl. Math. Adv. in Math. Supplementary Studies 18 (1983), 93-128.

11. __, On nonlinear Schrödinger equations, Ann. Inst. H. Poincaré. Phys. Théor. 46 (1987), 113-129.

12. A. K. Liu, Interactions of solitary waves in stratified fluids, Advances in Nonlinear Waves (L. Debnath, ed.), Vol. I, Research Notes in Mathematics, No. 95, Pitman, Boston, 1986, pp. 108-117.

13. P. Olver, Hamiltonian and nonhamiltonian models for water waves, Lecture Notes in Physics, no. 195, Springer-Verlag, Berlin and New York, 1984, pp. 273-290.

14. J. C. Saut, Sur quelques équations généralisant l'équation de Korteweg-de Vries, J. Math. Pures Appl. 58 (1979), 21-61.

15. P. Sjölin, Regularity of solutions to the Schrödinger equations, Uppsala University, Dept. of Math., Report no. 1986-14.

16. E. M. Stein, Singular integrals and dfferentiability properties of functions, Princeton Univ. Press, Princeton, N.J., 1970.

17. R. S. Strichartz, Restrictions of Fourier transforms to quadratic surfaces and decay of solutions of wave equations, Duke Math. J. 44 (1977), 705-714.

18. G. B. Whitham, Linear and nonlinear waves, Wiley, New York, 1974.

19. K. Yajima, Existence of solutions of Schrödinger evolution equations, Comm. Math. Phys. 110 (1987), 415-426.

Department of Mathematics, University of Chicago, Chicago, Illinois 60637

Université Paris XII and Laboratoire d'ANalyse Numérique, Université Paris-Sud, 91405 Orsay, France 